\title{
Soret and Dufour Effects on Unsteady Free Convection Fluid Flow in the Presence of Hall Current and Heat Flux
}

\author{
Abdul Quader1, Md. Mahmud Alam² \\ ${ }^{1}$ Department of Mathematics and Natural Sciences, School of Data and Sciences, BRAC University, Dhaka, Bangladesh \\ ${ }^{2}$ Mathematics Discipline, Science, Engineering and Technology School, Khulna University, Khulna, Bangladesh \\ Email: abdul.quader@bracu.ac.bd, alam_mahmud2000@yahoo.com
}

How to cite this paper: Quader, A. and Alam, Md.M. (2021) Soret and Dufour Effects on Unsteady Free Convection Fluid Flow in the Presence of Hall Current and Heat Flux. Journal of Applied Mathematics and Physics, 9, 1611-1638.

https://doi.org/10.4236/jamp.2021.97109

Received: June 16, 2021

Accepted: July 24, 2021

Published: July 27, 2021

Copyright $\odot 2021$ by author(s) and Scientific Research Publishing Inc. This work is licensed under the Creative Commons Attribution International License (CC BY 4.0).

http://creativecommons.org/licenses/by/4.0/

\begin{abstract}
Unsteady MHD natural convective heat and mass transfer flow through a semi-infinite vertical porous plate in a rotating system have been investigated with the combined Soret and Dufour effects in the presence of Hall current and constant heat flux. It is considered that the porous plate is subjected to constant heat flux. The obtained non-dimensional, non-similar coupled non-linear and partial differential equations have been solved by explicit finite difference technique. Numerical solutions for velocities, temperature and concentration distributions are obtained for various parameters by the above mentioned technique. The local and average shear stresses, Nusselt number as well as Sherwood number are also investigated. The stability conditions and convergence criteria of the explicit finite difference scheme are established for finding the restriction of the values of various parameters to get more accuracy. The obtained results are illustrated with the help of graphs to observe the effects of various legitimate parameters.
\end{abstract}

\section{Keywords}

Magnetohydrodynamic Flow, Heat and Mass Transfer, Hall Effect, Thermal Diffusion, Porous Medium, Finite Difference Technique

\section{Introduction}

The analysis of hydromagnetic natural convection flow involving heat and mass transfer in porous medium has attracted the attention of many scholars because of its possible applications in diverse fields of science and technology such as soil sciences, astrophysics, geophysics, nuclear power reactors, etc. In geophysics, it 
finds its applications in the design of MHD generators and accelerators, underground water energy storage system, etc. It is worth mentioning that MHD is now undergoing a stage of great enlargement and differentiation of subject matter. These new problems draw the attention of the researchers due to their varied significance in liquid metals, electrolytes and ionized gases, etc. The MHD in the present form is due to pioneer contributions of several notable authors like Crammer and Pai [1], Ferraro and Plumpton [2] and Shercliff [3]. Considerable attention has been given to the unsteady free-convection flow of viscous incompressible and electrically conducting fluid in the presence of applied magnetic field in connection with the theory of fluid motion in the liquid core of the earth, meteorological and oceanographic applications. The study of magnetohydrodynamic viscous flows with Hall currents has important engineering applications like Hall accelerators, power generators, constructions of turbines and centrifugal machines. In last few decades, the study of hydrodynamic and hydromagnetic boundary-layer flows with or without Hall current effects in a rotating fluid has received the attention of many research workers. Raptis et al. [4] have studied the effects of transverse magnetic field on hydromagnetic free convection considering Hall effects into account. Ram [5] investigated the effects of Hall current and wall temperature oscillation on convective flow in a rotating fluid through porous medium. The heat and mass transfer occur simultaneously between the fluxes, the driving potentials are of more intricate nature. An energy flux can be generated not only by temperature gradients but also by composition gradients. The energy flux caused by a composition is called Dufour or diffusion-thermo effect. Temperature gradients can also create mass fluxes and this is the Soret or thermal-diffusion effect. Generally, the thermal-diffusion and the diffusion-thermo effects are of smaller-order magnitude than the effects prescribed by Fourier's or Fick's laws and are often neglected in heat and mass transfer processes. However, there are exceptions. The thermal-diffusion effect, for instance, has been utilized for isotope separation and in mixture between gases with very light molecular weight (Hydrogen-Helium) and of medium molecular weight (Nitrogen-air), the diffusion-thermo effect was found to be of a magnitude such that it cannot be neglected. The boundary layer flows in the presence of Soret and Dufour effects associated with the thermal diffusion and diffusion-thermo for the mixed convection have been analyzed by Kafoussias and Williams [6]. The heat and mass transfer effects on a flow along a vertical plate in the presence of a magnetic field were investigated by Elbashbeshy [7]. The problem concerning MHD free convection and mass transfer flow with heat source and thermal diffusion was studied by Singh [8]. Takhar et al. [9] observed the MHD flow over a moving plate in a rotating fluid with magnetic field, Hall currents and free-stream velocity. The influence of porous media considering Soret and Dufour effects has been studied by Postelnicu [10]. Sharma et al. [11] have investigated the Hall effect on MHD mixed convective flow of a viscous incompressible fluid past a vertical porous plate immersed in a porous medium 
with heat source/sink. Wabomba et al. [12] investigated the effect of magnetic field and Hall current on MHD flow past a vertical rotating flat pate. The unsteady heat and mass transfer by mixed convection flow from a vertical porous plate with induced magnetic field, constant heat and mass fluxes has been investigated by Alam et al. [13]. The Hall effect on unsteady MHD flow past along a porous plate with thermal diffusion, diffusion thermo and chemical reaction has been investigated by Sudhakar et al. [14]. The effects of Hall currents, Soret and Dufour on MHD flow by mixed convection over vertical surface in porous media has been studied by Shateyi et al. [15].

In view of the significance of the Soret and Dufour effect as well as Hall current, it has been proposed in the present paper to investigate the unsteady MHD free convective flow past a vertical porous plate in porous medium with Hall current, thermal-diffusion, diffusion-thermo and heat flux. Here the main objectives are to study the effect of Dufour number, Soret number and Hall parameter on the flow and transport characteristics. The present study is an extension to the work done by Shateyi et al. [15] and to investigate the effects of both Hall current and constant heat flux with thermal diffusion and diffusion-thermo on an electrically conducting fluid bounded by a semi-infinite vertical porous plate in a rotating system. The proposed model has been transformed into non-similar coupled partial differential equation by usual transformations. The governing equations are solved numerically by using the explicit finite difference method with the help of a computer programming language Compaq Visual Fortran 6.6.a. Finally, the results of this study are discussed for different values of parameters and are shown graphically.

\section{Mathematical Analysis}

An unsteady flow model of combined heat and mass transfer by free convection of an electrically conducting incompressible viscous fluid past a semi-infinite vertical porous plate with the effects of Hall current and constant heat flux is considered which is illustrated in Figure 1.

Let the fluid rotate with uniform angular velocity $\Omega$ about the $z$-axis normal to the plate. It is assumed that there is a constant suction velocity. The flow is also assumed to be in the $x$-axis that is taken along the plate in the upward direction and $z$-axis is normal to it. At time $t>0$, the temperature at the plate and the species concentration are constantly raised from $T_{w}$ to $T_{\infty}$ and $C_{w}$ to $C_{\infty}$ respectively, which are thereafter maintained constant, where $T_{w}, C_{w}$ are the temperature and species concentration at the wall and $T_{\infty}, C_{\infty}$ are the temperature and species concentration far away from the plate respectively. A uniform magnetic field $H_{0}$ is imposed along the $z$-axis and the plate is taken to be electrically non-conducting. It is assumed that the induced magnetic field is negligible so that $\boldsymbol{H}=\left(0,0, H_{0}\right)$. This assumption is justified when the magnetic Reynolds number is very small. The equation of conservation of electric charge $\nabla \cdot \boldsymbol{J}=0$ gives $j_{z}=$ constant, where $\boldsymbol{J}=\left(j_{x}, j_{y}, j_{z}\right)$. This constant is 


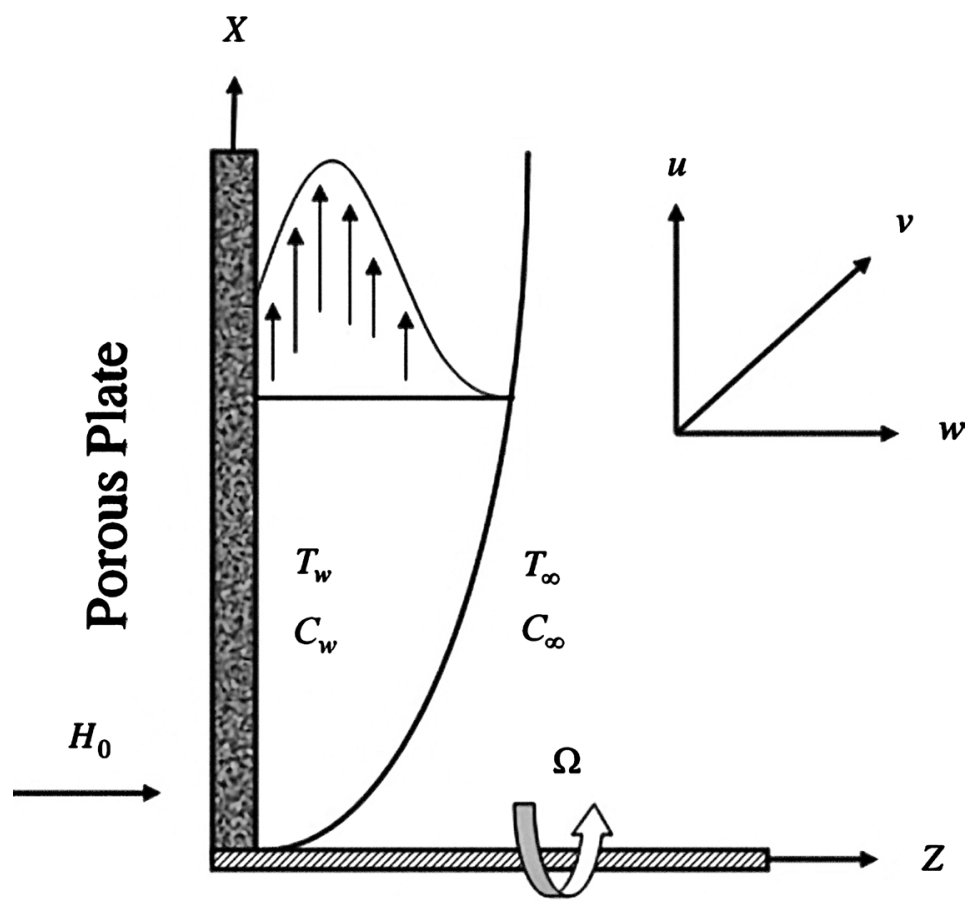

Figure 1. Physical configuration and coordinate system.

assumed to be zero at the non-conducting plate, therefore $j_{z}=0$ everywhere in the flow. The generalized Ohm's law including the effect of Hall current (Cowling [16]) is;

$$
\boldsymbol{J}+\frac{\omega_{e} \tau_{e}}{H_{0}}(\boldsymbol{J} \times \boldsymbol{H})=\sigma\left(\boldsymbol{E}+\mu_{e} \boldsymbol{q} \times \boldsymbol{H}+\frac{1}{e n_{e}} \nabla \cdot p_{e}\right)
$$

where $\omega_{e}$ is the cyclotron frequency and $\tau_{e}$ is electron collision time, $\sigma$ is the electric conductivity, $\mu_{e}$ is the magnetic permeability, $e$ is the electric charge, $n_{e}$ is the number density of electron and $p_{e}$ be the electron pressure. It has been assumed that the ion slip and thermoelectric effect is negligible. Further it is considered that the electric field $\boldsymbol{E}=0$ and electron pressure have been neglected. Under this assumption Equation (1) gives;

$$
\begin{aligned}
& j_{x}=\frac{\sigma \mu_{e} H_{0}}{1+m^{2}}(v+m u) \\
& j_{y}=\frac{\sigma \mu_{e} H_{0}}{1+m^{2}}(m v-u)
\end{aligned}
$$

Thus, accordance with the above assumptions relevant to the problem and under the electromagnetic Boussinesq approximation made by Ram [5], Sudhakar et al. [14] and Shateyi et al. [15] and in a rotating frame the basic boundary layer equations are given by;

The continuity equation

$$
\frac{\partial u}{\partial x}+\frac{\partial w}{\partial z}=0
$$

The momentum equations 


$$
\begin{gathered}
\frac{\partial u}{\partial t}+u \frac{\partial u}{\partial x}+w \frac{\partial u}{\partial z}= \\
g \beta\left(T-T_{\infty}\right)+g \beta^{*}\left(C-C_{\infty}\right)+v \frac{\partial^{2} u}{\partial z^{2}} \\
+v \frac{\sigma \mu_{e} H_{0}^{2}}{1+m^{2}}(m v-u)+2 \Omega v-\frac{v}{k^{\prime}} u \\
\frac{\partial v}{\partial t}+u \frac{\partial v}{\partial x}+w \frac{\partial v}{\partial z}=v \frac{\partial^{2} v}{\partial z^{2}}-v \frac{\sigma \mu_{e} H_{0}^{2}}{1+m^{2}}(v+m u)-2 \Omega u-\frac{v}{k^{\prime}} v
\end{gathered}
$$

\section{The energy equation}

$$
\begin{aligned}
\frac{\partial T}{\partial t}+u \frac{\partial T}{\partial x}+w \frac{\partial T}{\partial z}= & \frac{\kappa}{\rho C_{P}} \frac{\partial^{2} T}{\partial z^{2}}+\frac{v}{C_{P}}\left[\left(\frac{\partial v}{\partial z}\right)^{2}+\left(\frac{\partial u}{\partial z}\right)^{2}\right] \\
& +\frac{\sigma \mu_{e}^{2} H_{0}^{2}}{\rho C_{P}\left(1+m^{2}\right)}\left(u^{2}+v^{2}\right)+\frac{v}{C_{P}}\left[\left(\frac{\partial v}{\partial z}\right)^{2}+\left(\frac{\partial u}{\partial z}\right)^{2}\right] \\
& +\frac{\sigma \mu_{e}^{2} H_{0}^{2}}{\rho C_{P}\left(1+m^{2}\right)}\left(u^{2}+v^{2}\right)+\frac{Q}{\rho C_{P}}\left(T_{\infty}-T\right)+\frac{D_{m} \kappa_{T}}{C_{s} C_{p}} \frac{\partial^{2} C}{\partial z^{2}}
\end{aligned}
$$

The species equation

$$
\frac{\partial C}{\partial t}+u \frac{\partial C}{\partial x}+w \frac{\partial C}{\partial z}=D_{m} \frac{\partial^{2} C}{\partial z^{2}}+\frac{D_{m} \kappa_{T}}{T_{m}} \frac{\partial^{2} T}{\partial z^{2}}
$$

with the corresponding initial and boundary conditions are;

$$
\begin{gathered}
t=0, u=0, v=0, w=0, T \rightarrow T_{\infty}, C \rightarrow C_{\infty} \quad \text { everywhere } \\
t>0, u=0, v=0, w=0, T \rightarrow T_{\infty}, C \rightarrow C_{\infty} \quad \text { at } x=0 \\
u=U_{0}, v=0, w=0, \frac{\partial T}{\partial z}=-\frac{Q}{\kappa}, C=C_{w} \quad \text { at } z=0 \\
u=0, v=0, w=0, T \rightarrow T_{\infty}, C \rightarrow C_{\infty} \quad \text { as } z \rightarrow \infty
\end{gathered}
$$

where $u, v$ and $w$ are the $x, y$ and $z$ components of velocity vector respectively, $m=\omega_{e} \tau_{e}$ is the Hall parameter, $v$ is the coefficient of kinematic viscosity, $\rho$ is the density of the fluid, $\mu_{e}$ be the magnetic permeability, $\sigma$ is the electrical conductivity, $H_{0}$ be the uniform magnetic field, $U_{0}$ be the uniform velocity, $g$ is the acceleration due to gravity, $\beta$ is the coefficient of thermal expansion, $\beta^{*}$ is the coefficient of concentration expansion, $k^{\prime}$ is the permeability of the porous medium, $\kappa$ is thermal conductivity, $C_{P}$ is the specific heat at constant pressure, $C_{s}$ be the concentration susceptibility, $Q$ be the constant heat flux per unit area, $D_{m}$ be the coefficient of mass diffusivity, $\kappa_{T}$ be the thermal diffusion ratio and $T_{m}$ be the mean fluid temperature.

To obtain the governing equations and the boundary conditions in dimensionless form, the following non-dimensional quantities are introduced as;

$$
\begin{aligned}
& X=\frac{x U_{0}}{v}, Z=\frac{z U_{0}}{v}, U=\frac{U}{U_{0}}, V=\frac{V}{U_{0}}, W=\frac{W}{U_{0}}, \\
& \tau=\frac{t U_{0}^{2}}{v}, \theta=\frac{\kappa U_{0}\left(T-T_{\infty}\right)}{Q v} \text { and } \varphi=\frac{C-C_{\infty}}{C_{w}-C_{\infty}}
\end{aligned}
$$

Substituting the above relations in Equations (4)-(8) and after simplification, the following non-linear coupled partial differential equations in terms of di- 
mensionless variables are obtained as;

$$
\begin{gathered}
\frac{\partial U}{\partial X}+\frac{\partial W}{\partial Z}=0 \\
\frac{\partial U}{\partial \tau}+U \frac{\partial U}{\partial X}+W \frac{\partial U}{\partial Z}=G_{r} \theta+G_{m} \varphi+\frac{\partial^{2} U}{\partial Z^{2}}+\frac{M}{1+m^{2}}(m V-U)+2 E_{k} V-\frac{U}{k} \\
\frac{\partial V}{\partial \tau}+U \frac{\partial V}{\partial X}+W \frac{\partial V}{\partial Z}=\frac{\partial^{2} V}{\partial Z^{2}}-\frac{M}{1+m^{2}}(V+m U)-2 E_{k} U-\frac{V}{k} \\
\frac{\partial \theta}{\partial \tau}+U \frac{\partial \theta}{\partial X}+W \frac{\partial \theta}{\partial Z} \\
\frac{1}{P_{r}} \frac{\partial^{2} \theta}{\partial Z^{2}}+E_{c}\left[\left(\frac{\partial V}{\partial Z}\right)^{2}+\left(\frac{\partial U}{\partial Z}\right)^{2}\right]+\frac{M E_{c}}{1+m^{2}}\left(U^{2}+V^{2}\right)-\frac{\alpha}{P_{r}} \theta+D_{f} \frac{\partial^{2} \varphi}{\partial Z^{2}} \\
\frac{\partial \varphi}{\partial \tau}+U \frac{\partial \varphi}{\partial X}+W \frac{\partial \varphi}{\partial Z}=\frac{1}{S_{c}} \frac{\partial^{2} \varphi}{\partial Z^{2}}+S_{r} \frac{\partial^{2} \theta}{\partial Z^{2}}
\end{gathered}
$$

The corresponding initial and boundary conditions in Equations (9) and (10) become;

$$
\begin{gathered}
\tau>0, U=0, V=0, W=0, \theta=0, \varphi=0 \text { at } X=0 \\
U=1, V=0, W=0, \frac{\partial \theta}{\partial Z}=-1, \varphi=1 \text { at } Z=0 \\
U=0, V=0, W=0, \theta=0, \varphi=0 \text { at } Z \rightarrow \infty
\end{gathered}
$$

where, $G_{r}=\frac{g \beta Q v^{2}}{\kappa U_{0}^{4}}$ (Grashoff number), $G_{m}=\frac{v g \beta^{*}\left(C_{w}-C_{\infty}\right)}{U_{0}^{3}}$ (Modified Grashoff number), $E_{k}=\frac{\Omega v}{U_{0}^{2}}$ (Ekman number), $M=\frac{\sigma \mu_{e}^{2} H_{0}^{2} v}{\rho U_{0}^{2}}$ (Magnetic parameter), $k=\frac{U_{0}^{2} k^{\prime}}{v^{2}}$ (Permeability parameter), $E_{c}=\frac{\kappa U_{0}^{3}}{Q v C_{p}}$ (Eckert number), $P_{r}=\frac{v \rho C_{p}}{\kappa}$ (Prandtl number), $S_{c}=\frac{v}{D_{m}}$ (Schmidt number), $\alpha=\frac{Q v^{2}}{\kappa U_{0}^{2}}$ (Heat source parameter), $D_{f}=\frac{D_{m} \kappa_{T}}{C_{s} C_{p}} \frac{U_{0} \kappa}{Q v^{2}}\left(C_{w}-C_{\infty}\right)$ (Dufour number), $S_{r}=\frac{D_{m} \kappa_{T}}{T_{m}} \frac{Q}{\kappa U_{0}\left(C_{w}-C_{\infty}\right)}$ (Soret number).

\section{Shear Stresses, Nusselt Number and Sherwood Number}

From the velocity field, the effects of various parameters on local and average shear stresses have been studied. The following equations represent the local and average shear stresses at the plate. The local primary shear stress is in the $x$-direction, $\tau_{x_{L}}=\mu\left(\frac{\partial u}{\partial z}\right)_{z=0}$ and average primary shear stress is in the $x$-direction, $\tau_{x_{A}}=\mu \int\left(\frac{\partial u}{\partial z}\right)_{Z=0} d x$ which are proportional to $\left(\frac{\partial U}{\partial Z}\right)_{Z=0}$ and $\int_{0}^{100}\left(\frac{\partial U}{\partial Z}\right)_{Z=0} d X$ 
respectively. The local secondary shear stress is in the $y$-direction, $\tau_{y_{L}}=\mu\left(\frac{\partial v}{\partial z}\right)_{z=0}$ and average primary shear stress is in the $y$-direction, $\tau_{y_{A}}=\mu \int\left(\frac{\partial v}{\partial z}\right)_{z=0} \mathrm{~d} x$ which are proportional to $\left(\frac{\partial V}{\partial Z}\right)_{Z=0}$ and $\int_{0}^{100}\left(\frac{\partial V}{\partial Z}\right)_{Z=0} \mathrm{~d} X$ respectively. From the temperature field, the effects of various parameters on heat transfer coefficient have been calculated. The following equation represents the local and average heat transfer rate, which is well known as Nusselt number. Local Nusselt number, $N_{u_{L}}=\mu\left(-\frac{\partial T}{\partial z}\right)_{z=0}$ and average Nusselt number, $N_{u_{A}}=\mu \int\left(-\frac{\partial T}{\partial z}\right)_{z=0} \mathrm{~d} x$ which are proportional to $\left(-\frac{\partial \theta}{\partial Z}\right)_{Z=0}$ and $\int_{0}^{100}\left(-\frac{\partial \theta}{\partial Z}\right)_{Z=0} \mathrm{~d} X$ respectively. From the concentration field, the effects of various parameters on mass transfer coefficient have been calculated. The following equation represents the local and average mass transfer rate, which is well known as Sherwood number. Local Sherwood number, $S_{h_{L}}=\mu\left(-\frac{\partial C}{\partial z}\right)_{z=0}$ and average Sherwood number, $S_{h_{A}}=\mu \int\left(-\frac{\partial C}{\partial z}\right)_{z=0} \mathrm{~d} x$

which are proportional to $\left(-\frac{\partial \varphi}{\partial Z}\right)_{Z=0}$ and $\int_{0}^{100}\left(-\frac{\partial \varphi}{\partial Z}\right)_{Z=0} \mathrm{~d} X$ respectively.

\section{Numerical Technique}

To solve the above non-dimensional system of equations by explicit finite difference technique, the present problem requires a set of finite difference equations. To obtain the difference equations the region within the boundary layer is divided into a grid or mesh lines parallel to $X$ and $Z$-axes where $X$-axis is taken along the plate and $Z$-axis is normal to the plate. It is considered that the plate of height $X_{\max }(=100)$ and regard $Z_{\max }(=25)$ as corresponding to $Z \rightarrow \infty$. There are $m=180$ and $n=180$ grid spacing in $X$ and $Z$ directions respectively as shown in Figure 2. It is assumed that $\Delta X, \Delta Z$ are constant mesh sizes along $X$ and $Z$ directions respectively and taken as follows

$\Delta X=0.555(0 \leq X \leq 100)$ and $\Delta Z=0.138(0 \leq X \leq 25)$ with a smaller time space $\Delta \tau=0.005$.

The explicit finite difference approximation gives;

$$
\begin{gathered}
\frac{U_{i, j}^{n+1}-U_{i-1, j}^{n+1}}{\Delta X}+\frac{W_{i, j}^{n}-W_{i, j-1}^{n}}{\Delta Z}=0 \\
\frac{U_{i, j}^{n+1}-U_{i, j}^{n}}{\Delta \tau}+U_{i, j}^{n} \frac{U_{i, j}^{n}-U_{i-1, j}^{n}}{\Delta X}+W_{i, j}^{n} \frac{U_{i, j+1}^{n}-U_{i, j}^{n}}{\Delta Z} \\
=G_{r} \theta_{i, j}^{n}+G_{m} \varphi_{i, j}^{n}+\frac{U_{i, j+1}^{n}-2 U_{i, j}^{n}+U_{i, j-1}^{n}}{(\Delta Z)^{2}} \\
+\frac{M}{1+m^{2}}\left(m V_{i, j}^{n}-U_{i, j}^{n}\right)+2 E_{k} V_{i, j}^{n}-\frac{U_{i, j}^{n}}{k}
\end{gathered}
$$




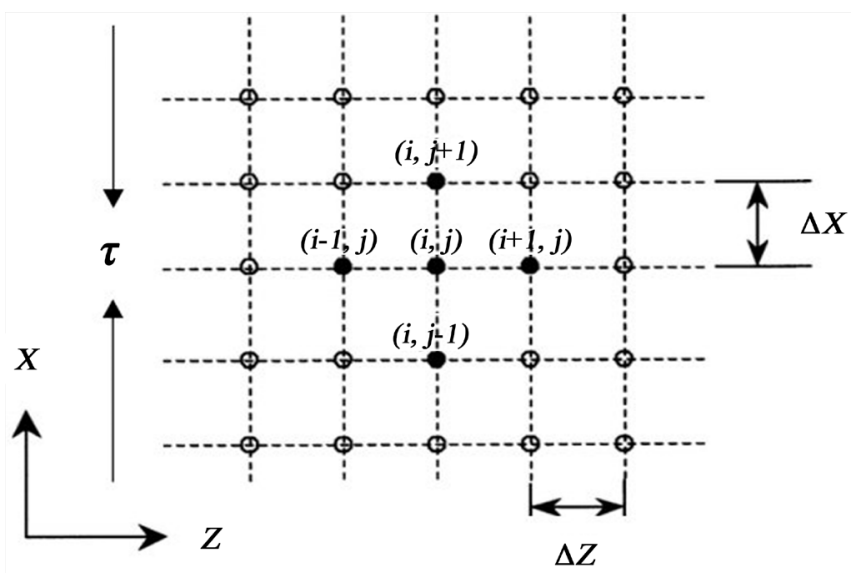

Figure 2. Explicit finite difference space grid.

$$
\begin{aligned}
& \frac{V_{i, j}^{n+1}-V_{i, j}^{n}}{\Delta \tau}+U_{i, j}^{n} \frac{V_{i, j}^{n}-V_{i-1, j}^{n}}{\Delta X}+W_{i, j}^{n} \frac{V_{i, j+1}^{n}-V_{i, j}^{n}}{\Delta Z} \\
& =\frac{V_{i, j+1}^{n}-2 V_{i, j}^{n}+V_{i, j-1}^{n}}{(\Delta Z)^{2}}-\frac{M}{1+m^{2}}\left(V_{i, j}^{n}+m U_{i, j}^{n}\right)-2 E_{k} U_{i, j}^{n}-\frac{V_{i, j}^{n}}{k} \\
& \frac{\theta_{i, j}^{n+1}-\theta_{i, j}^{n}}{\Delta \tau}+U_{i, j}^{n} \frac{\theta_{i, j}^{n}-\theta_{i-1, j}^{n}}{\Delta X}+W_{i, j}^{n} \frac{\theta_{i, j+1}^{n}-\theta_{i, j}^{n}}{\Delta Z} \\
& =\frac{1}{P_{r}} \frac{\theta_{i, j+1}^{n}-2 \theta_{i, j}^{n}+\theta_{i, j-1}^{n}}{(\Delta Z)^{2}}+\frac{M E_{c}}{1+m^{2}}\left(\left(U_{i, j}^{n}\right)^{2}+\left(V_{i, j}^{n}\right)^{2}\right) \\
& +E_{c}\left[\left(\frac{V_{i, j+1}^{n}-V_{i, j}^{n}}{\Delta Z}\right)^{2}+\left(\frac{U_{i, j+1}^{n}-U_{i, j}^{n}}{\Delta Z}\right)^{2}\right] \\
& \quad-\frac{\alpha}{P_{r}} \theta_{i, j}^{n}+D_{f} \frac{\varphi_{i, j+1}^{n}-2 \varphi_{i, j}^{n}+\varphi_{i, j-1}^{n}}{(\Delta Z)^{2}} \\
& \quad \frac{\varphi_{i, j}^{n+1}-\varphi_{i, j}^{n}}{\Delta \tau}+U_{i, j}^{n} \frac{\varphi_{i, j}^{n}-\varphi_{i-1, j}^{n}}{\Delta X}+W_{i, j}^{n} \frac{\varphi_{i, j+1}^{n}-\varphi_{i, j}^{n}}{\Delta Z} \\
& =\frac{1}{S_{c}} \frac{\varphi_{i, j+1}^{n}-2 \varphi_{i, j}^{n}+\varphi_{i, j-1}^{n}}{(\Delta Z)^{2}}+S_{r} \frac{\theta_{i, j+1}^{n}-2 \theta_{i, j}^{n}+\theta_{i, j-1}^{n}}{(\Delta Z)^{2}}
\end{aligned}
$$

with the finite difference boundary conditions ;

$$
\begin{gathered}
U_{0, j}^{n}=0, V_{0, j}^{n}=0, \theta_{0, j}^{n}=0, \varphi_{0, j}^{n}=0 \\
U_{i, 0}^{n}=1, V_{i, 0}^{n}=0, \frac{\theta_{i, 1}^{n}-\theta_{i, 0}^{n}}{\Delta Z}=-1, \varphi_{i, 0}^{n}=1 \\
U_{i, L}^{n}=0, V_{i, L}^{n}=0, \theta_{i, L}^{n}=0, \varphi_{i, L}^{n}=0 \text { where } L \rightarrow \infty
\end{gathered}
$$

Here the subscript $i$ and $j$ designates the grid points with $X$ and $Z$ coordinate and $n$ represents a value of time, $\tau=n \Delta \tau$ where $n=1,2,3, \cdots$. At the end of time step $\Delta \tau$, the primary velocity, the secondary velocity, the temperature distributions and the concentration distributions at all interior nodal points, may be calculated by successive applications of the above finite difference equa- 
tions. Also the numerical values of the local shear stresses, Nusselt number and Sherwood number are evaluated by Five-point approximate formula and then the average shear stresses, Nusselt number and Sherwood number are calculated by the use of the Simpson's one-third integration formula.

\section{Stability and Convergence Analysis}

Here Since an explicit procedure is being used, the analysis will remain incomplete unless we discuss the stability and convergence of the finite difference scheme. For the constant mesh sizes, the stability criteria of the scheme may be established as follows;

$$
\begin{gathered}
U \frac{\Delta \tau}{\Delta X}-|W| \frac{\Delta \tau}{\Delta Z}+\frac{2 \Delta \tau}{(\Delta Z)^{2}}+\left(\frac{M}{1+m^{2}}+\frac{1}{k}\right) \frac{\Delta \tau}{2} \leq 1 \\
U \frac{\Delta \tau}{\Delta X}-|W| \frac{\Delta \tau}{\Delta Z}+\frac{1}{P_{r}} \frac{2 \Delta \tau}{(\Delta Z)^{2}}+\frac{\alpha}{P_{r}} \frac{\Delta \tau}{2} \leq 1 \\
U \frac{\Delta \tau}{\Delta X}-|W| \frac{\Delta \tau}{\Delta Z}+\frac{1}{S_{c}} \frac{2 \Delta \tau}{(\Delta Z)^{2}} \leq 1
\end{gathered}
$$

Form the above Equations (23)-(25), the convergence limits for the model of flow are $M \leq 89.9, m \geq 0.001, k \geq 0.01, \alpha \leq 5.99, P_{r} \geq 0.54$ and $S_{c} \geq 0.526$.

\section{Results and Discussion}

To investigate the practical situation of the problem, the approximate solutions are obtained for various parameters. In order to analyze the physical situation of the model, the steady state numerical values of the dimensionless primary velocity $(U)$, secondary velocity $(V)$, temperature $(\theta)$ and concentration $(\varphi)$ within the boundary layer for different values of Hall parameter $(m)$, Magnetic parameter $(M)$, Heat source parameter $(\alpha)$, Grashof number $\left(G_{r}\right)$, porous permeability parameter $(k)$, Ekman number $\left(E_{k}\right)$, Eckert number $\left(E_{c}\right)$, Prandtl number $\left(P_{r}\right)$, Schmidt number $\left(S_{c}\right)$, Dufour number $\left(D_{f}\right)$ and Soret number $\left(S_{r}\right)$ with the fixed value of modified Grashof number $\left(G_{m}\right)$ have been obtained. To compute the numerical results, a computer programming language Compaq Visual Fortran 6.6.a has been used and Tecplot 9.0 is used for displaying figures graphically.

For the steady state solutions of the problem, the computations have been carried out up to $\tau=80$. It is observed that the values of this computation, however, show little changes after $\tau=60$. Thus the solution at $\tau=60$ are essentially steady-state solutions. Because of the great importance of cooling problem in nuclear engineering in connection with the cooling of reactors, the value of the Grashof number for heat transfer is taken positive $\left(G_{r}>0\right)$. Since the most important fluids are atmospheric air, salt water and water so the results are limited to $P_{r}=0.71$ (Prandtl number for air at $20^{\circ} \mathrm{C}$ ), $P_{r}=1.0$ (Prandtl number for salt water at $20^{\circ} \mathrm{C}$ ) and $P_{r}=7.0$ (Prandtl number for water at $20^{\circ} \mathrm{C}$ ). Here the investigation are assumed for both lighter and heavier fluid particles, hence with respect to convergence criteria of the problem the values of Schmidt number $\left(S_{c}\right)$ 
are taken $0.60,0.78$ and 0.97 (in particular, 0.60 for water vapor that represents a diffusing chemical species of most common interest in air, 0.78 corresponds to ammonia and 0.97 for carbon dioxide which represents the specific condition of the flow). In addition, The values of Hall parameter $(m)$, Magnetic parameter $(M)$, Heat source parameter $(\alpha)$ and porous permeability parameter $(k)$ are taken according to the convergence criteria. However, the values of other parameters $E_{k}, E_{\mathcal{c}}, D_{f}$ and $S_{r}$ are chosen arbitrarily and also the modified Grashof number $G_{m}=1.0$ for mass transfer are considered arbitrarily.

Along with the obtained steady state solutions, the flow behaviors in case of cooling problem are discussed graphically. The nature of primary velocity, secondary velocity, temperature distributions and concentration distributions versus $Z$ are illustrated in Figures 3-30.

The primary velocity profiles have been shown in Figures 3-8. The effect of heat source parameter $(\alpha)$ on the primary velocity $(U)$ field is presented in Figure 3. It is observed that $U$ decreases with the increase of $\alpha$. The primary velocity has an increasing effect for the rise of Hall parameter $(m)$, which is presented in Figure 4. It is analyzed that the primary velocity decreases with the rise of magnetic parameter $(M)$ which is plotted in Figure 5. It is observed in Figure 6 that the primary velocity rapidly increases with the increase of Eckert number $\left(E_{c}\right)$. It is observed from Figure 7 that the primary velocity $(U)$ field decreases near the plate in case of rising Dufour number $\left(D_{f}\right)$, but far away from the plate the increase of $D_{f}$ leads to an increase in primary velocity field that is indicating a cross flow of primary velocity for $D_{f}$. Figure 8 depicts the Soret effect on primary velocity $(U)$ field and it shows that the velocity rises with the increase of Soret number $\left(S_{r}\right)$.

Figures 9-14 represent the secondary velocity profiles for cooling plate $\left(G_{r}>0\right)$. The effect of heat source parameter $(\alpha)$ on the secondary velocity $(V)$ field is presented in Figure 9. It is observed that $V$ increases with the increase of $\alpha$. The secondary velocity profile decreases significantly for the rise of Hall

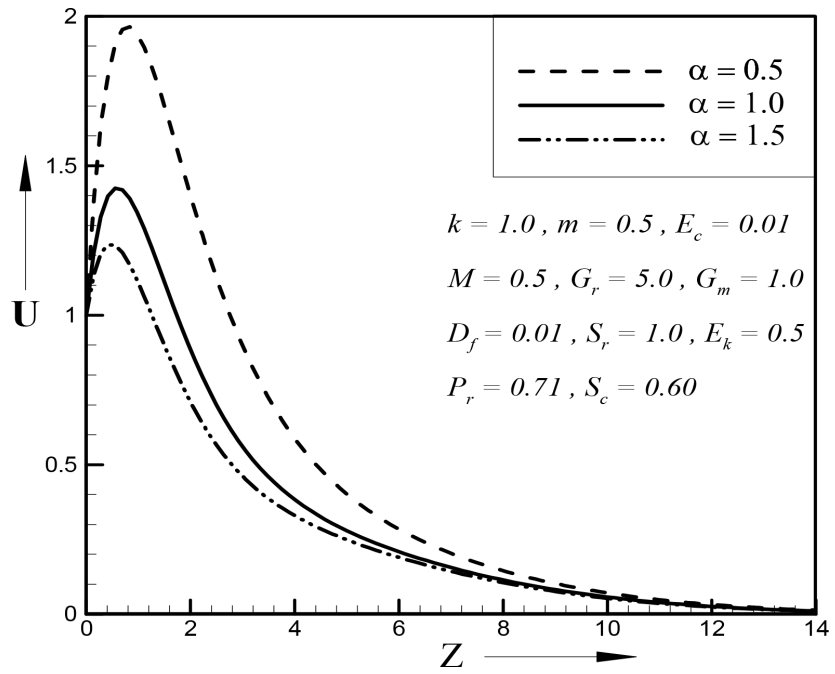

Figure 3. Primary velocity profiles for different values of $\alpha$. 


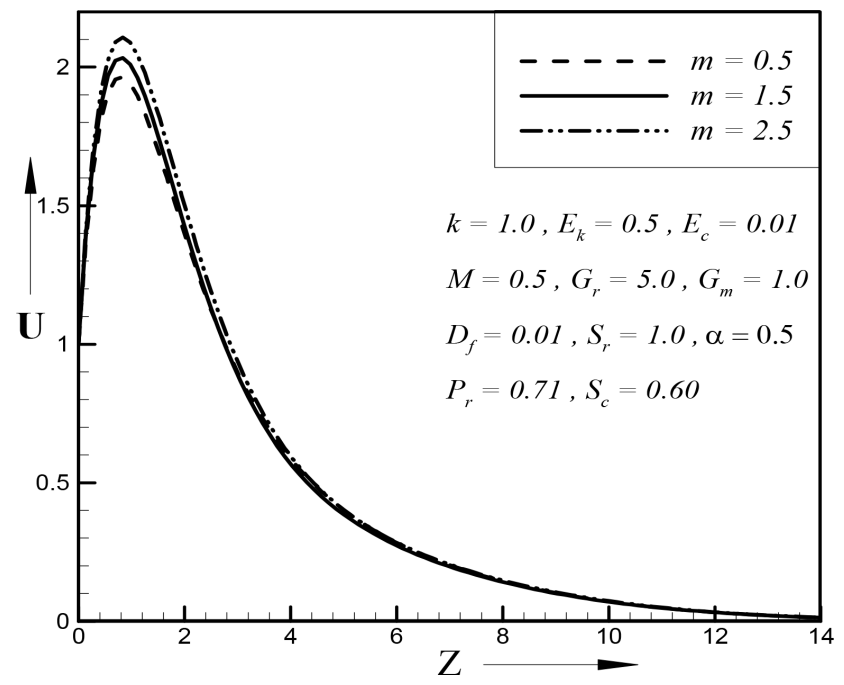

Figure 4. Primary velocity profiles for different values of $m$.

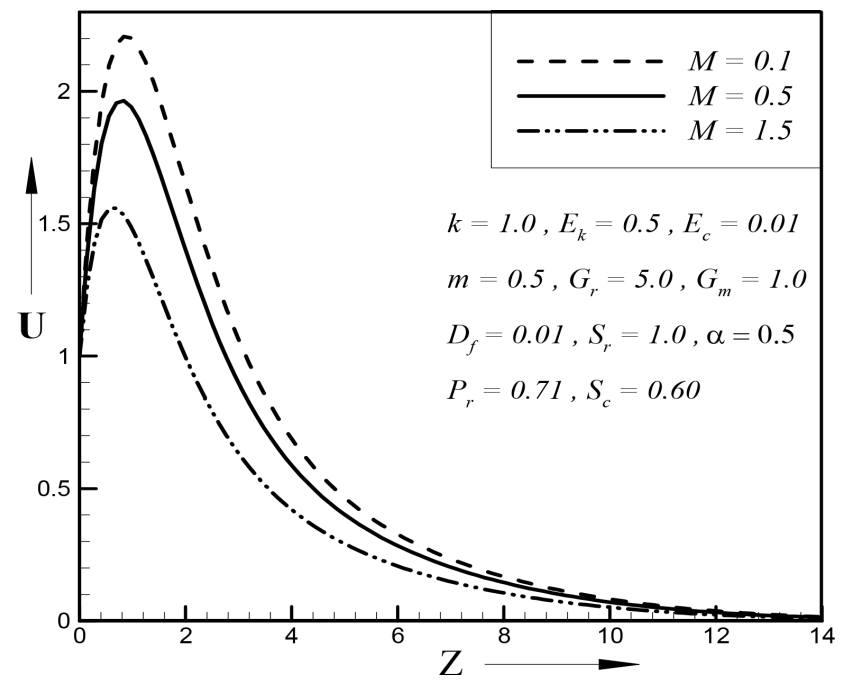

Figure 5. Primary velocity profiles for different values of $M$.

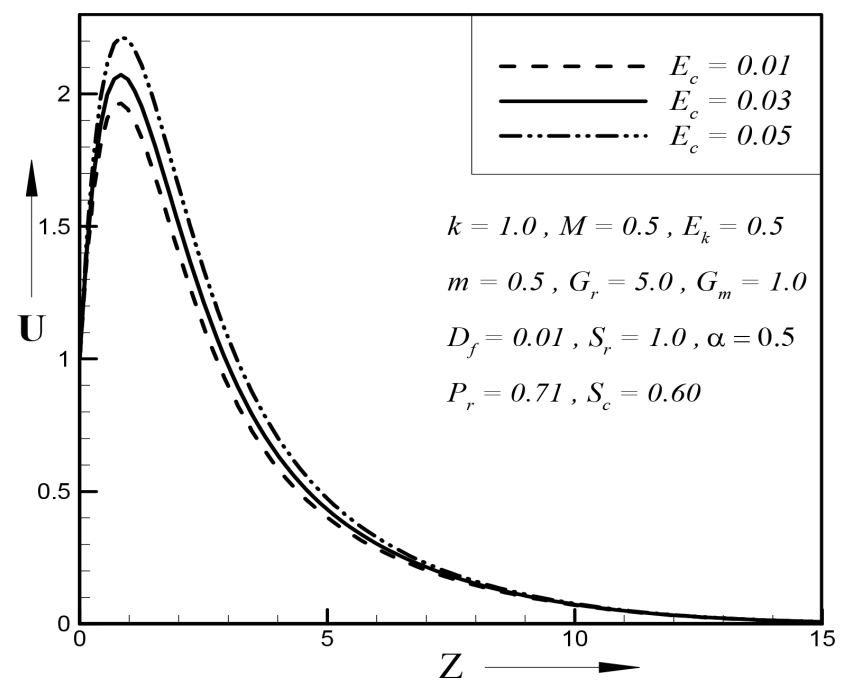

Figure 6. Primary velocity profiles for different values of $E_{c}$. 


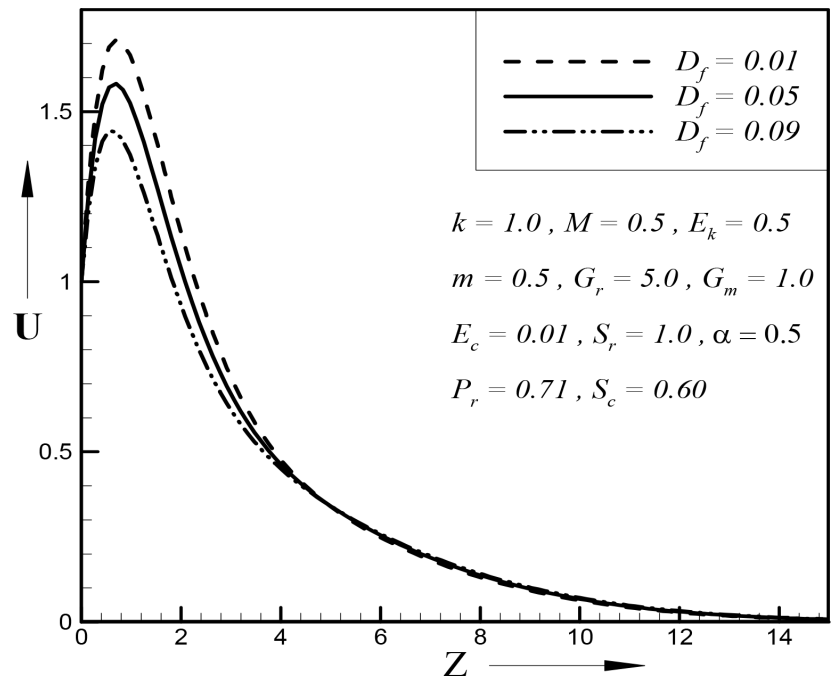

Figure 7. Primary velocity profiles for different values of $D_{f}$.

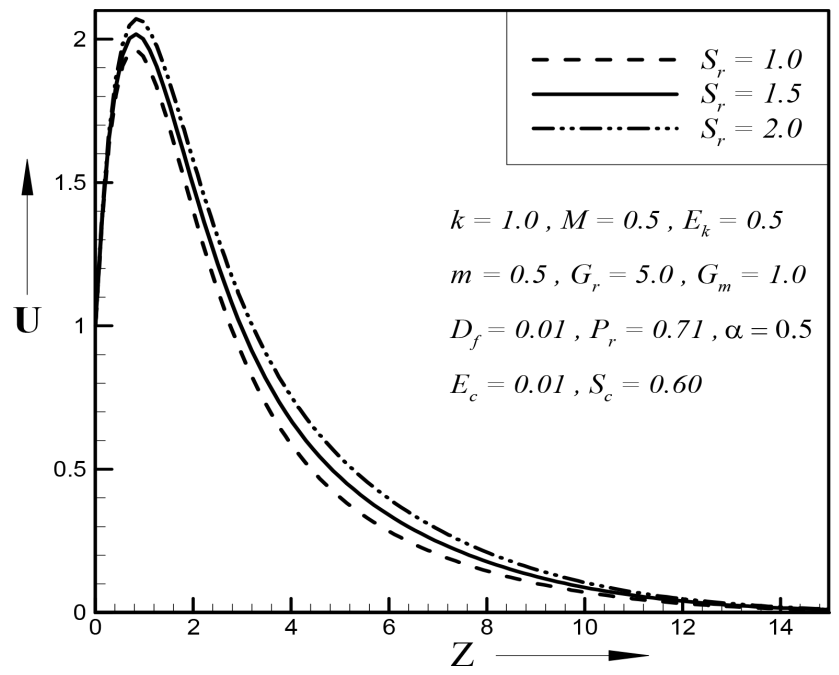

Figure 8. Primary velocity profiles for different values of $S_{r}$.

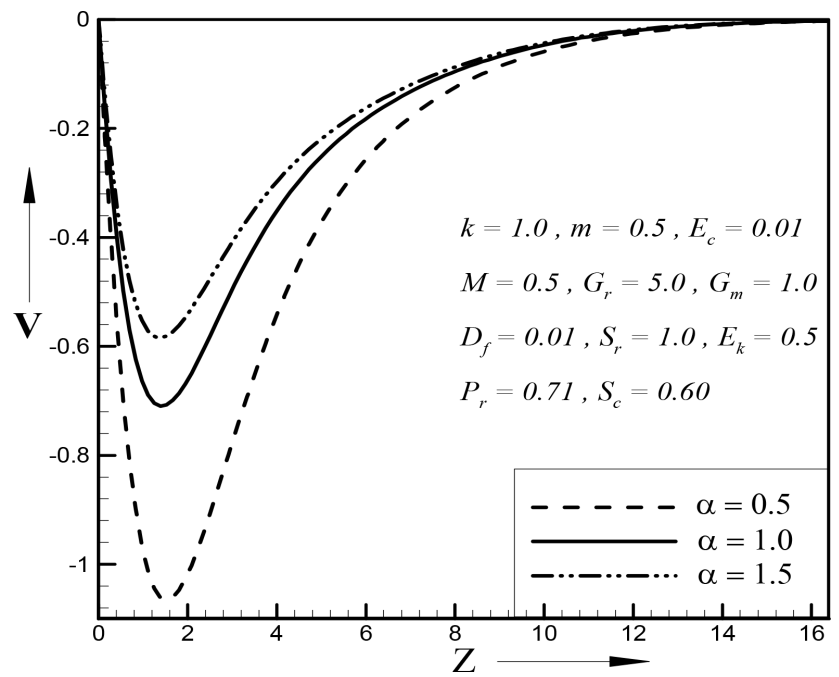

Figure 9. Secondary velocity profiles for different values of $\alpha$. 


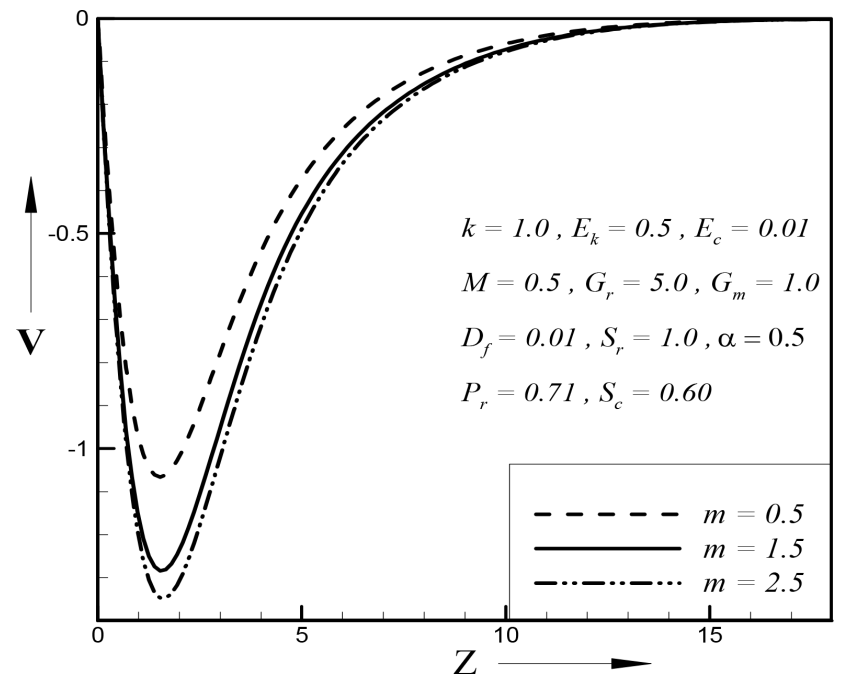

Figure 10. Secondary velocity profiles for different values of $m$.

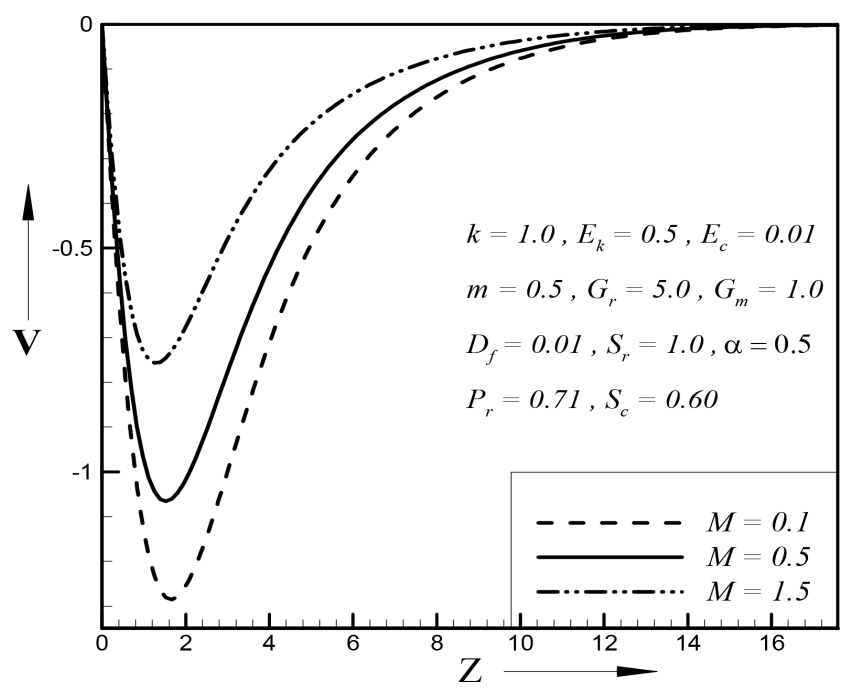

Figure 11. Secondary velocity profiles for different values of $M$.

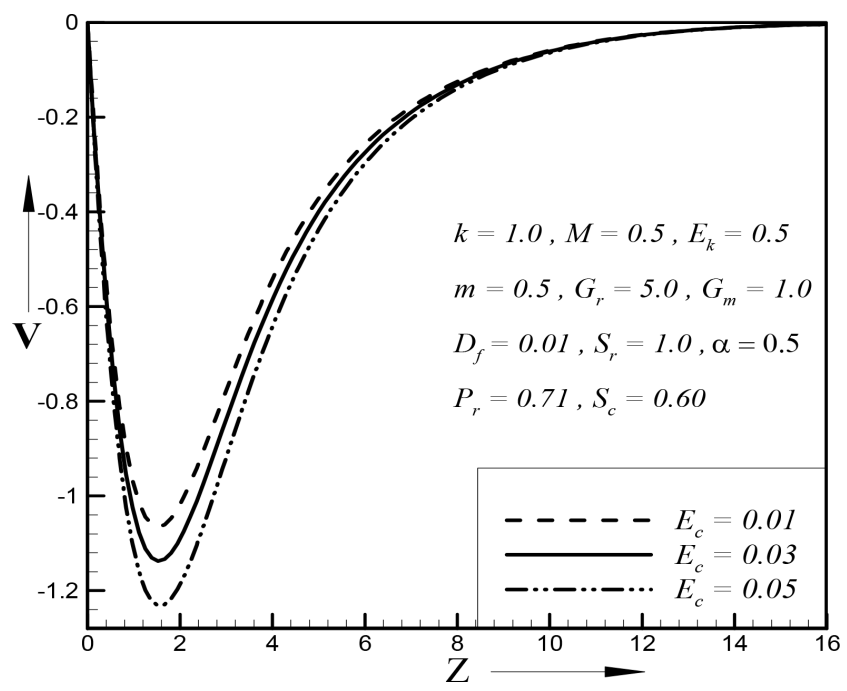

Figure 12. Secondary velocity profiles for different values of $E_{c}$. 


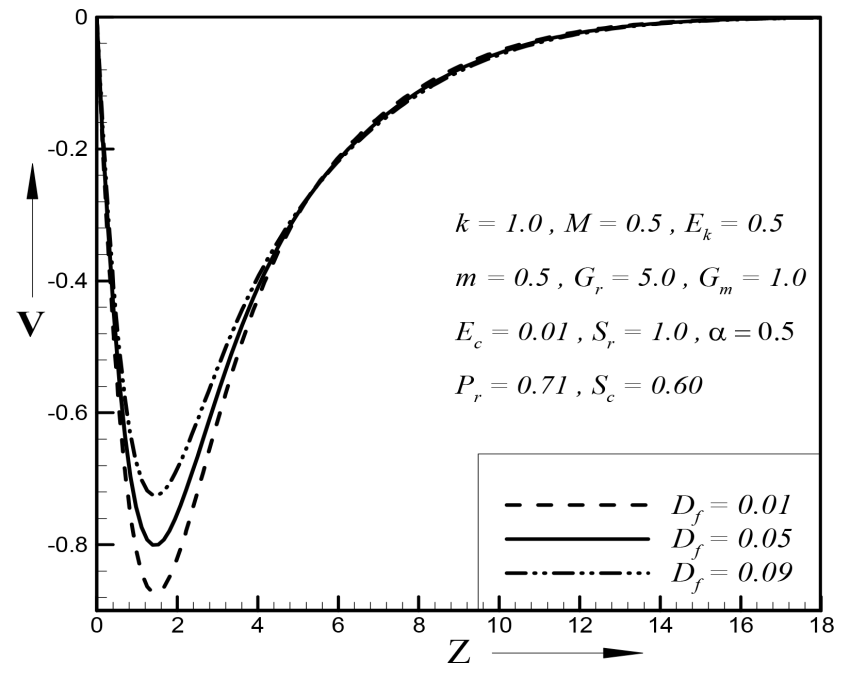

Figure 13. Secondary velocity profiles for different values of $D_{f}$.

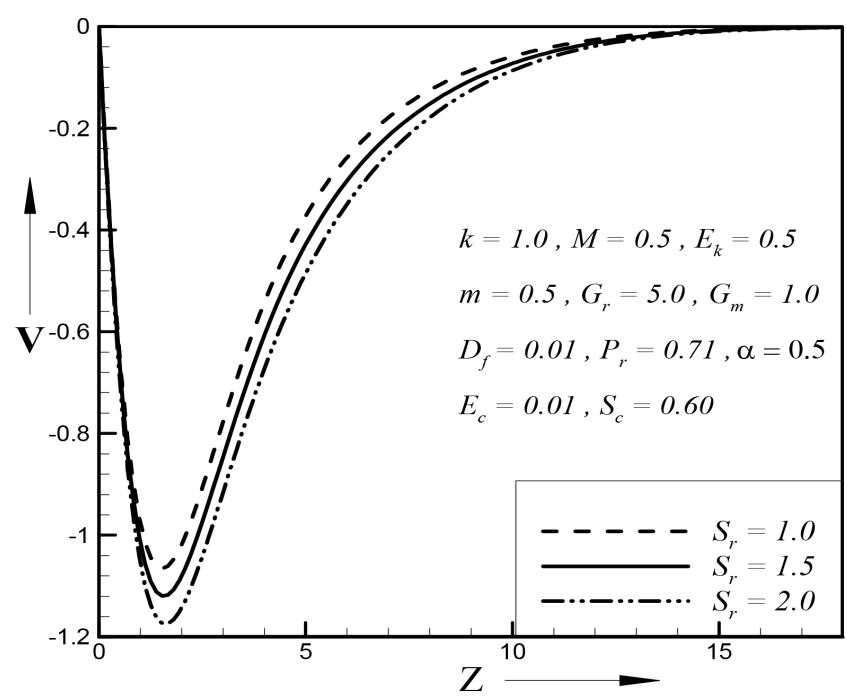

Figure 14. Secondary velocity profiles for different values of $S_{r}$.

parameter $(m)$, which is presented in Figure 10. It is analyzed that the secondary velocity increases with the increase of magnetic parameter $(M)$, which is plotted in Figure 11. It is observed in Figure 12 that the secondary velocity decreases with the increase of Eckert number $\left(E_{c}\right)$. Figure 13 depicts the secondary velocity $(V)$ field increases near the plate in case of rising Dufour number $\left(D_{f}\right)$, but far away from the plate the increase of $D_{f}$ leads to a decrease in secondary velocity field that is indicating a cross flow of secondary velocity for $D_{f}$ Figure 14 illustrates the Soret effect on secondary velocity $(V)$ field and it shows that the velocity decreases with the increase of Soret number $\left(S_{r}\right)$. Specially, it is observed that the velocity distribution increases or decreases gradually near the plate and then decreases or increases slowly far away from the plate. Hence, it is concluded that the maximum velocity occurs in the vicinity of the plate.

The temperature distributions are shown in Figures 15-20. It is observed in Figure 15 that the temperature profile decreases with the increase of heat source 


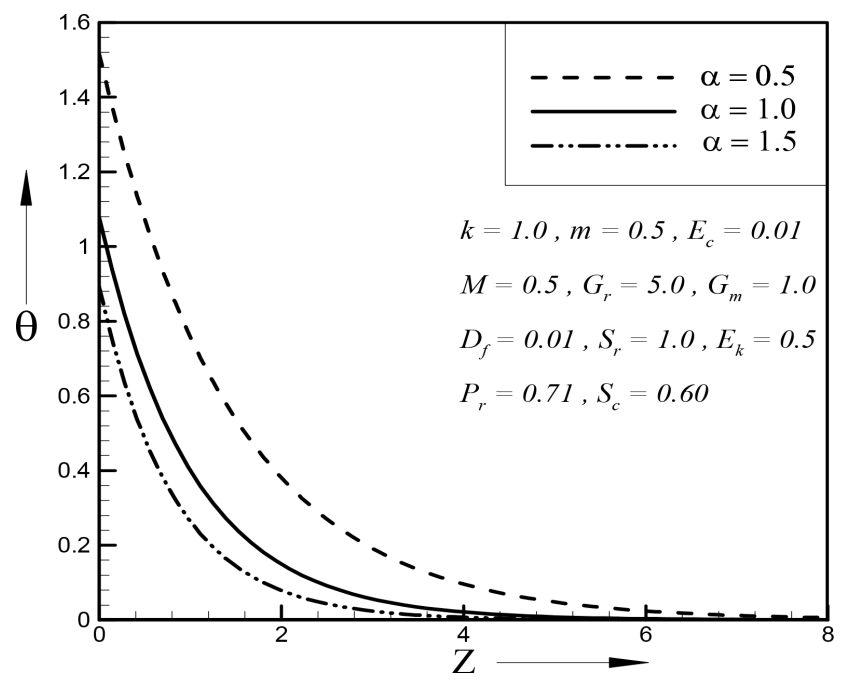

Figure 15. Temperature profiles for different values of $\alpha$.

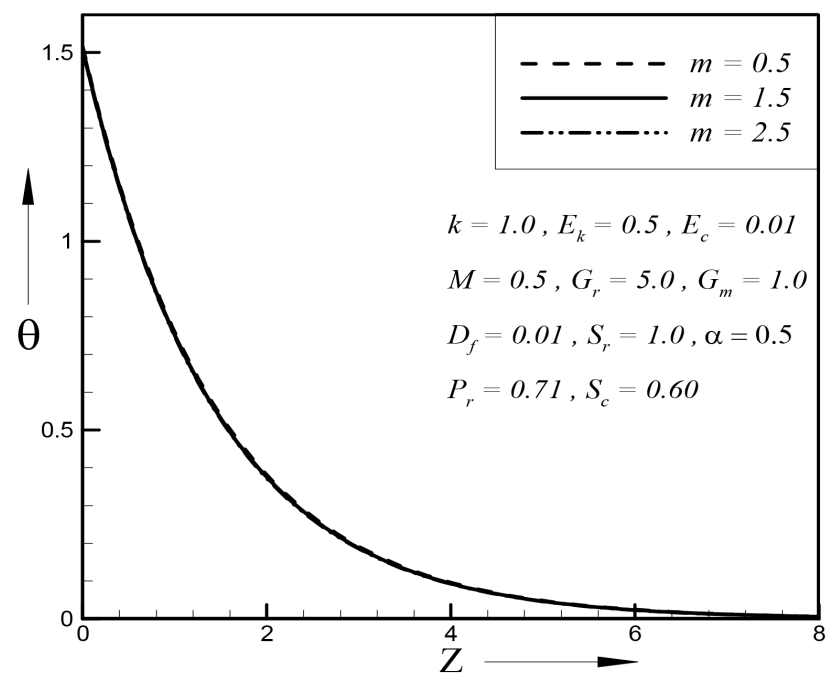

Figure 16. Temperature profiles for different values of $m$.

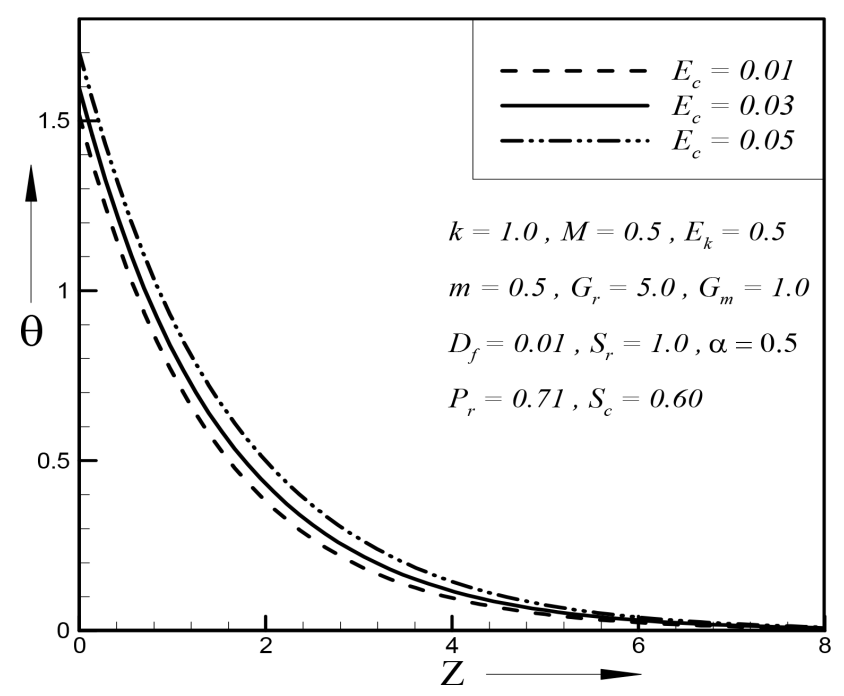

Figure 17. Temperature profiles for different values of $E_{c}$. 


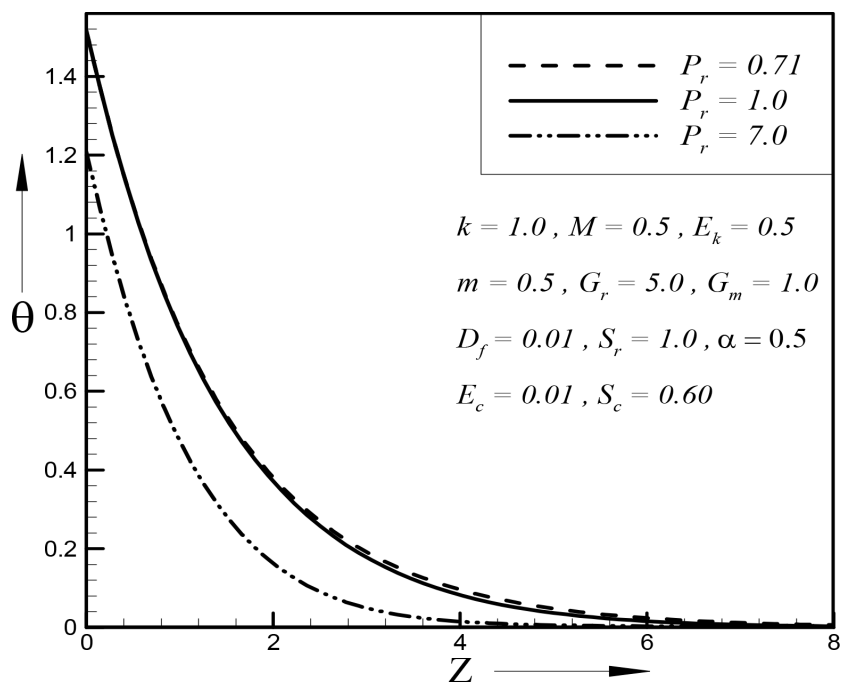

Figure 18. Temperature profiles for different values of $P_{r}$.

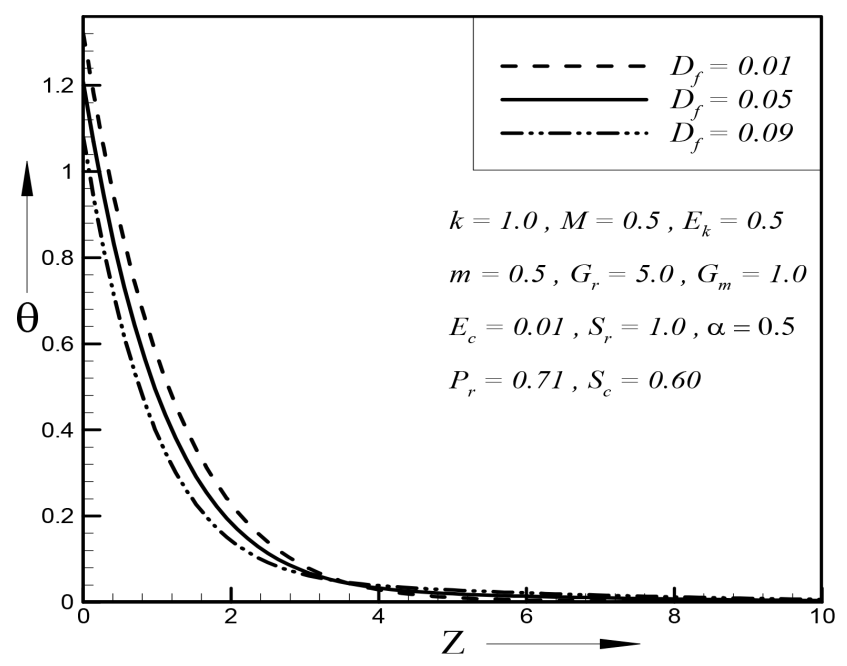

Figure 19. Temperature profiles for different values of $D_{f}$.

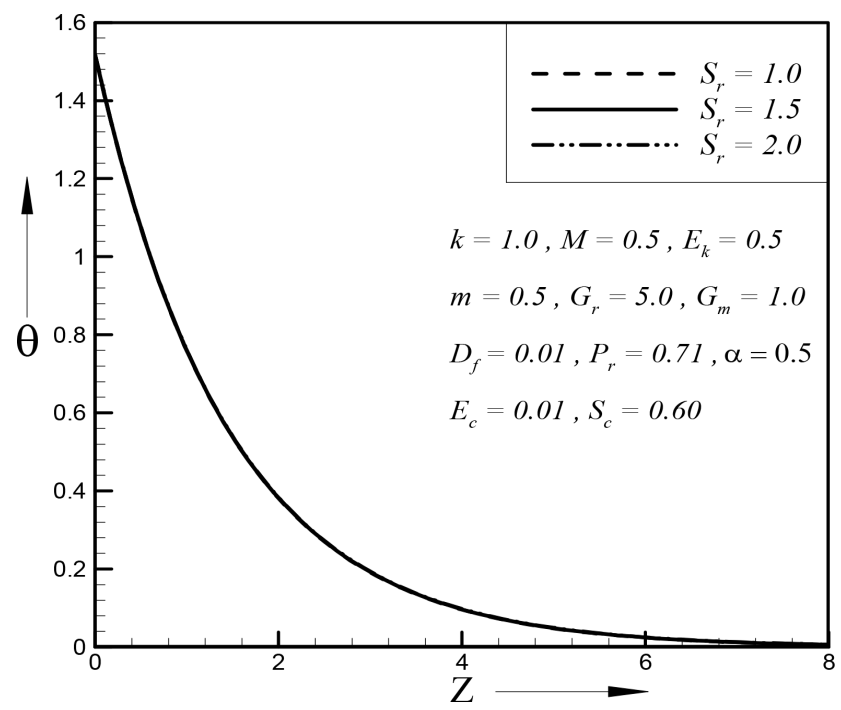

Figure 20. Temperature profiles for different values of $S_{r}$. 
parameter $(\alpha)$. Figure 16 shows that Hall parameter $(m)$ has a minor effect on temperature $(\theta)$ distribution. From Figure 17, it is shown that the increasing values of the Eckert number $\left(E_{c}\right)$ increases the temperature distribution significantly. Figure 18 shows that the temperature decreases drastically for the increase of Prandtl number $\left(P_{r}\right)$. This is due to the fact that there would be a decrease of thermal boundary layer thickness for the increase of $P_{r}$. It is observed in Figure 19 that the temperature $(\theta)$ profile decreases near the plate for rising Dufour number $\left(D_{f}\right)$, but it increases far away from the plate for $D_{f}$ that is indicating a cross flow of temperature distribution for $D_{f}$ Figure 20 is illustrated that the temperature distribution has a minor increasing effect for Soret number $\left(S_{r}\right)$.

The species concentration distributions are presented in Figures 21-24. It is observed in Figure 21 that the concentration decreases with the increase of heat

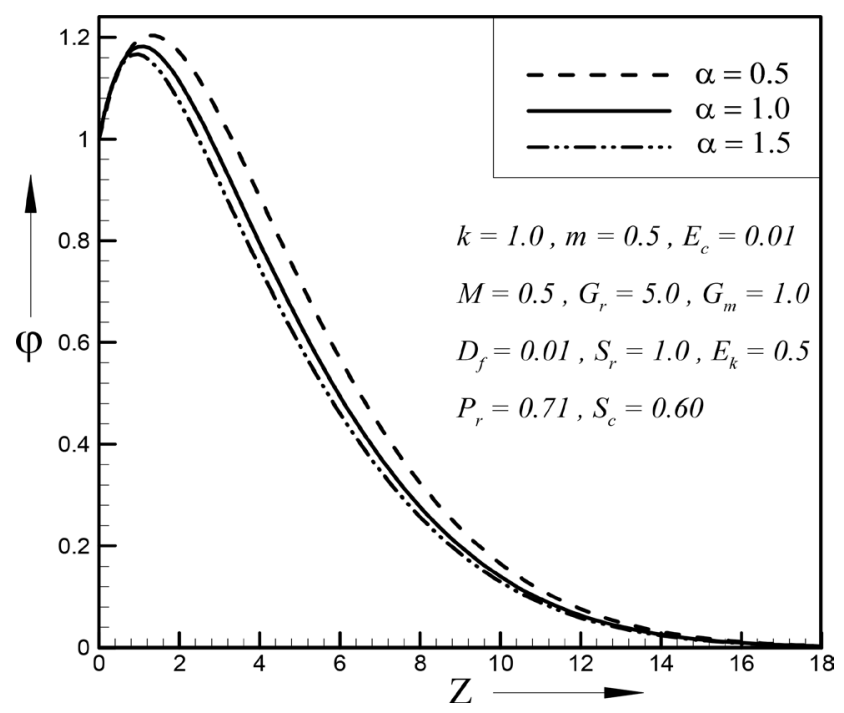

Figure 21. Concentration profiles for different values of $\alpha$.

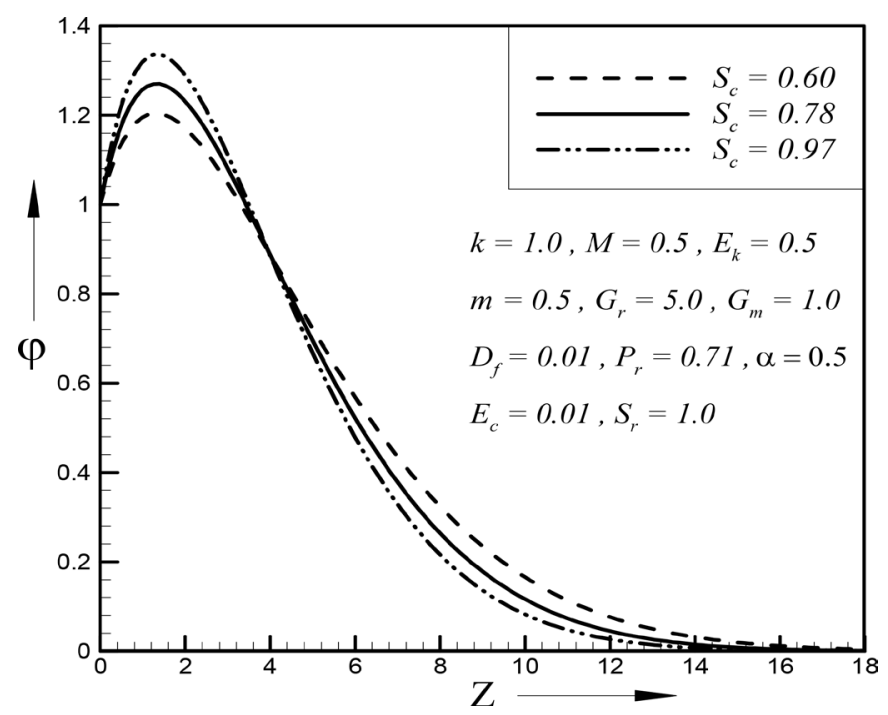

Figure 22. Concentration profiles for different values of $S_{c}$ 


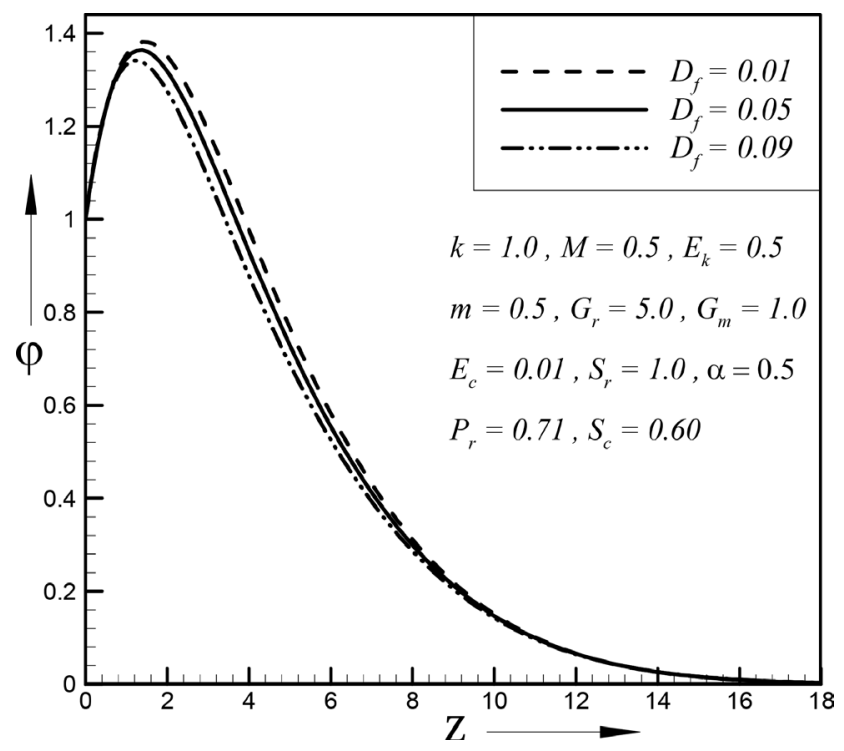

Figure 23. Concentration profiles for different values of $D_{f}$.

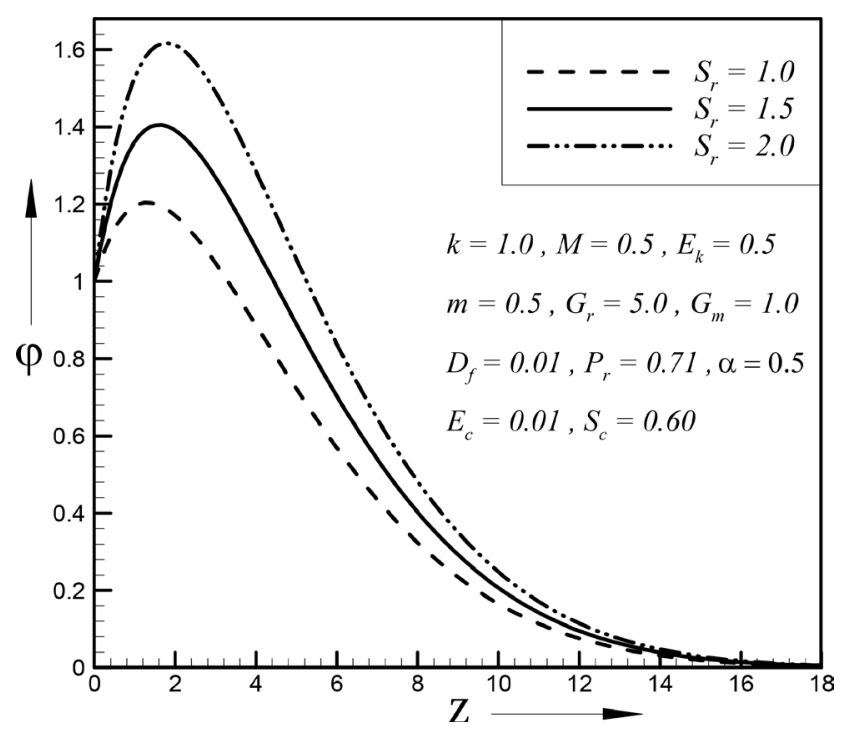

Figure 24. Concentration profiles for different values of $S_{r}$.

source parameter $(\alpha)$. The increase of Schmidt number $\left(S_{c}\right)$ shows an increasing effect on concentration profile near the plate and then it gradually decreases far away from the plate that means there exists a cross flow for $S_{c}$ which is observed in Figure 22. Physically, the increase of $S_{c}$ means the decrease of molecular diffusivity. Hence the concentration of species is higher for small values of $S_{c}$ and lower for large values of $S_{c}$. It is observed in Figure 23 that the concentration $(\varphi)$ profile decreases near the plate for rising Dufour number $\left(D_{f}\right)$, but it increases far away from the plate for the increase of $D_{f}$ that is indicating a cross flow of concentration distribution for $D_{f}$ Figure 24 depicts that the concentration $(\varphi)$ rises with the increase of Soret number $\left(S_{r}\right)$.

The steady state local and average primary shear stresses $\tau_{x_{L}}$ and $\tau_{x_{A}}$ are shown in Figures 25-30. It is observed in Figures 25-26 that, $\tau_{x_{L}}$ and $\tau_{x_{A}}$ 


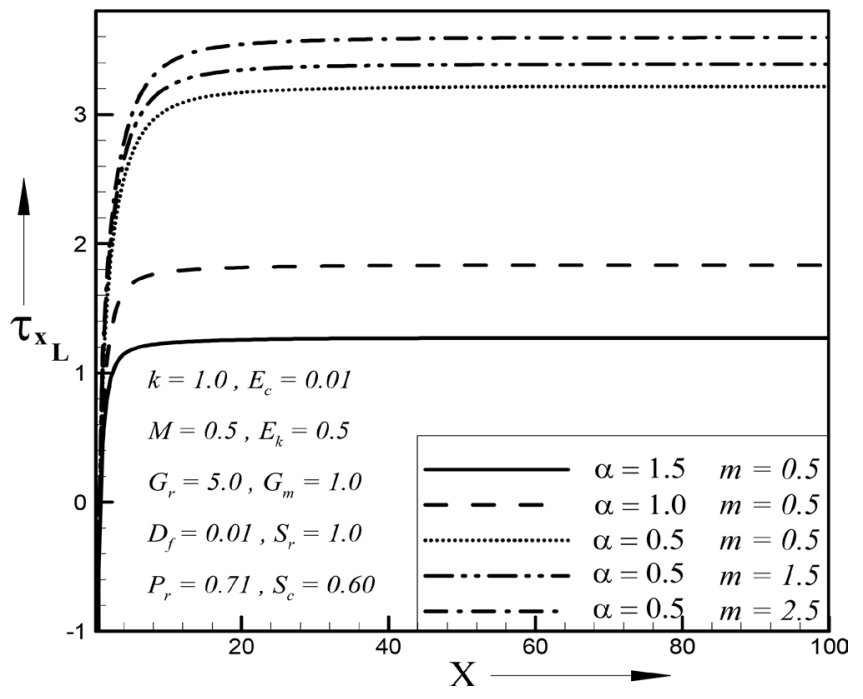

Figure 25. Local primary shear stress for different values of $\alpha$ and $m$.

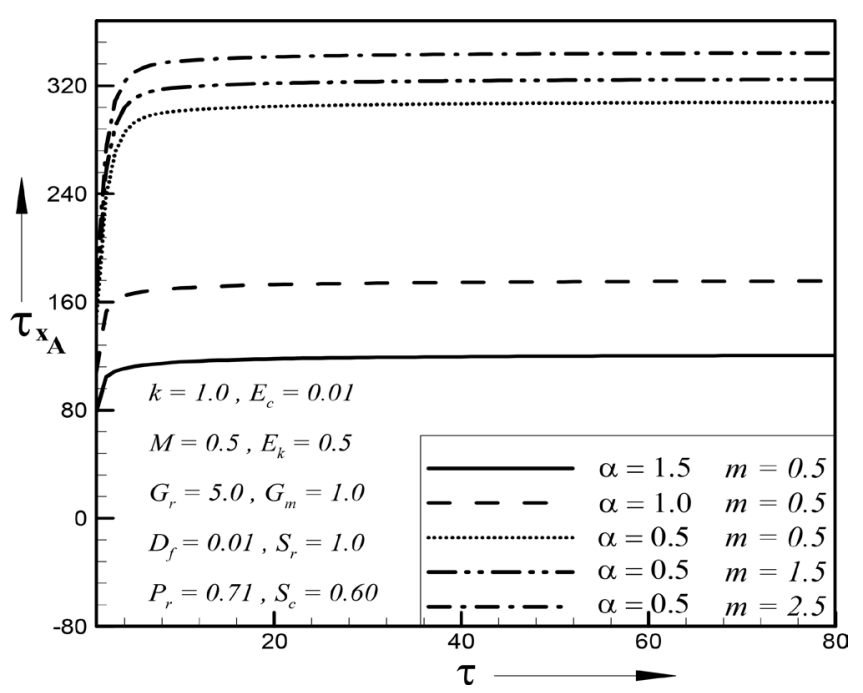

Figure 26. Average primary shear stress for different values of $\alpha$ and $m$.

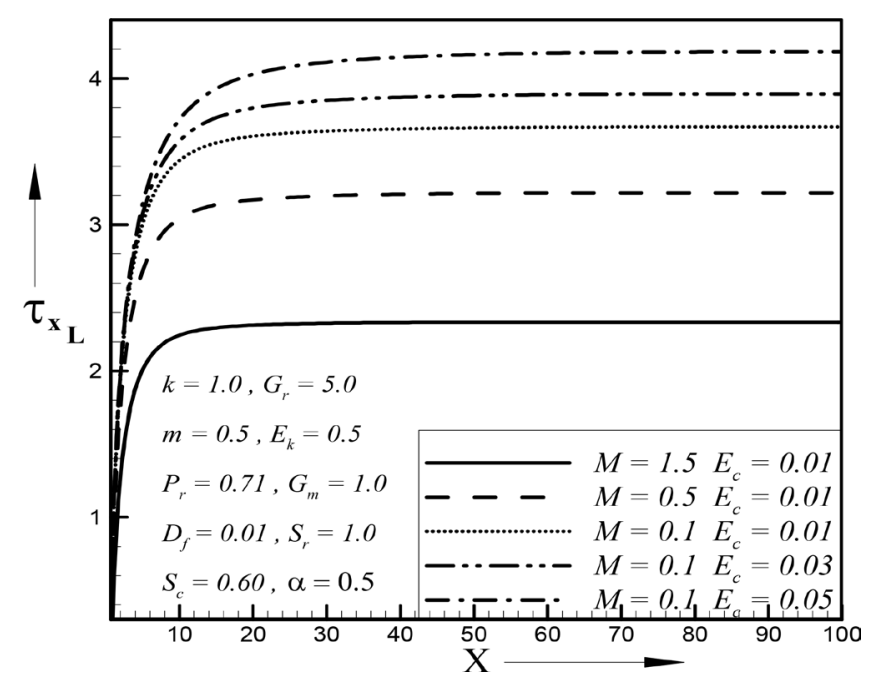

Figure 27. Local primary shear stress for different values of $M$ and $E_{c}$. 


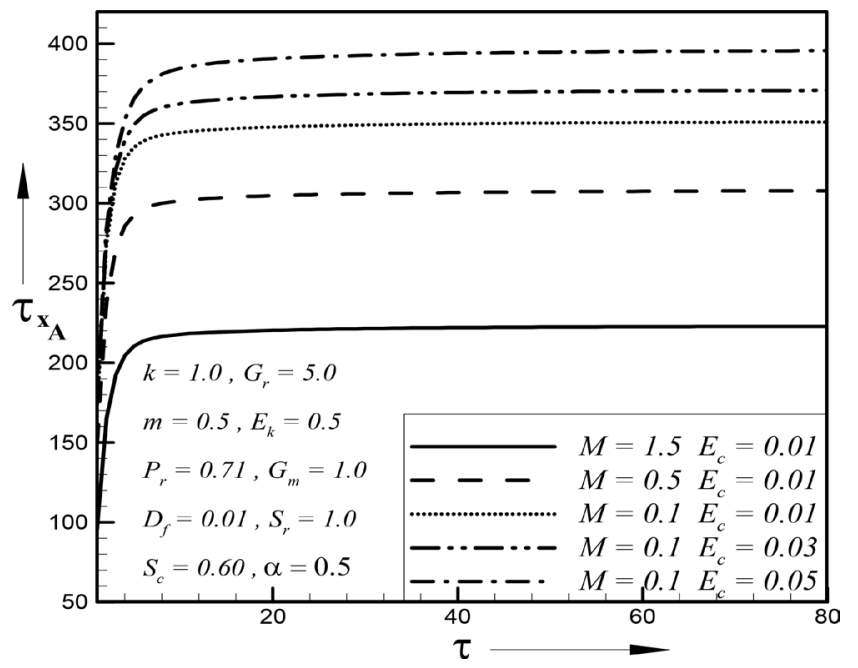

Figure 28. Average primary shear stress for different values of $M$ and $E_{c}$.

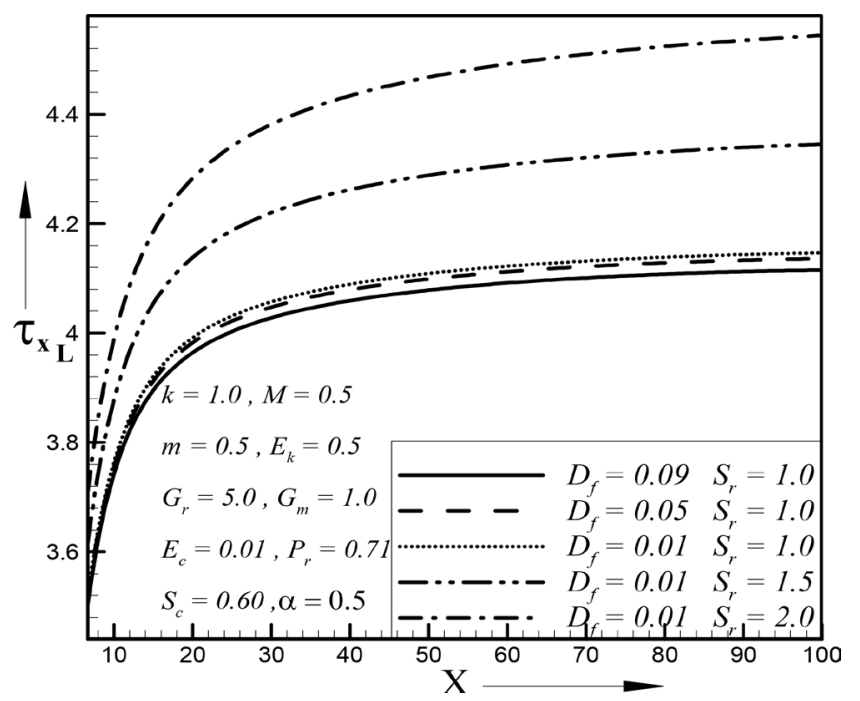

Figure 29. Local primary shear stress for different values of $D_{f}$ and $S_{r}$.

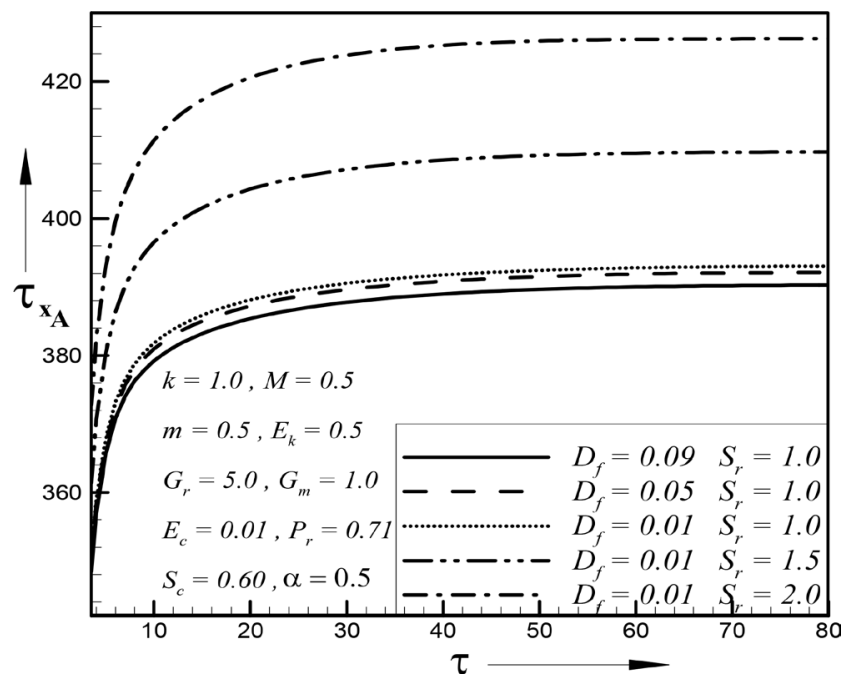

Figure 30. Average primary shear stress for different values of $D_{f}$ and $S_{I}$. 
decrease with the increase of Heat source parameter $(\alpha)$ while they both have an increasing effect for the increase of Hall parameter $(m)$. The local and average primary shear stresses decrease with the increase of magnetic parameter $(M)$ while they both increase with the increase of Eckert number $\left(E_{c}\right)$, which are plotted in Figure 27 \& Figure 28. Local and average primary shear stresses are decreasing for increasing Dufour number $\left(D_{f}\right)$ while for the increase of Soret number $\left(S_{r}\right)$, they are increasing those are observed in Figure 29 \& Figure 30.

In Figures 31-36, the steady state local and average secondary shear stress $\tau_{y_{L}}$ and $\tau_{y_{A}}$ are presented for different values of parameters. It is observed in both Figure 31 \& Figure 32 that, $\tau_{y_{L}}$ and $\tau_{y_{A}}$ increase with the increase of Heat source parameter $(\alpha)$ while they have a decreasing effect for the rise of Hall parameter $(m)$. It is analyzed that the local and average secondary shear stresses increases with the increase of magnetic parameter $(M)$ while they both decrease with the increase of Eckert number $\left(E_{c}\right)$, which is plotted in Figure 33 \& Figure 34.

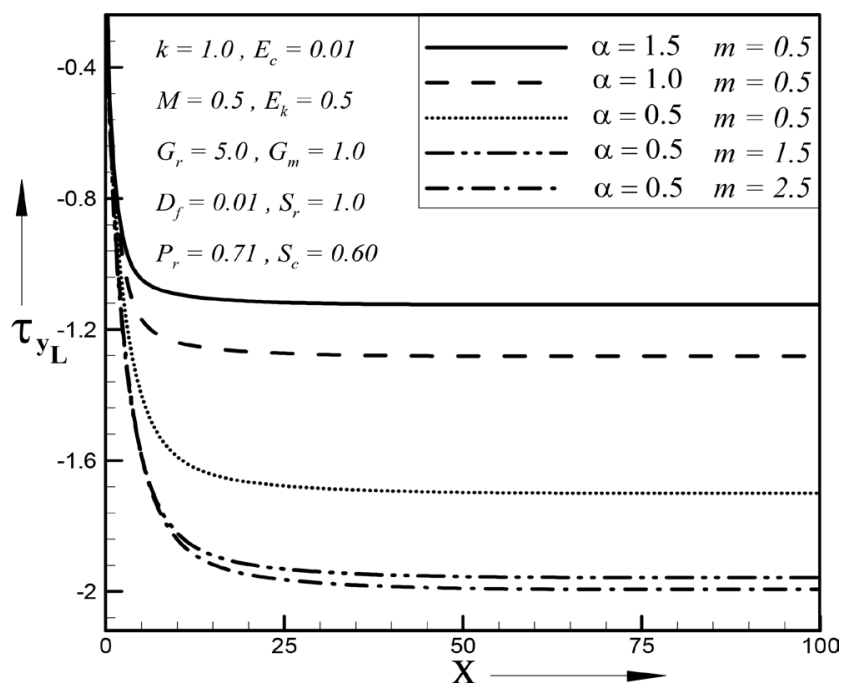

Figure 31. Local secondary shear stress for different values of $\alpha$ and $m$.

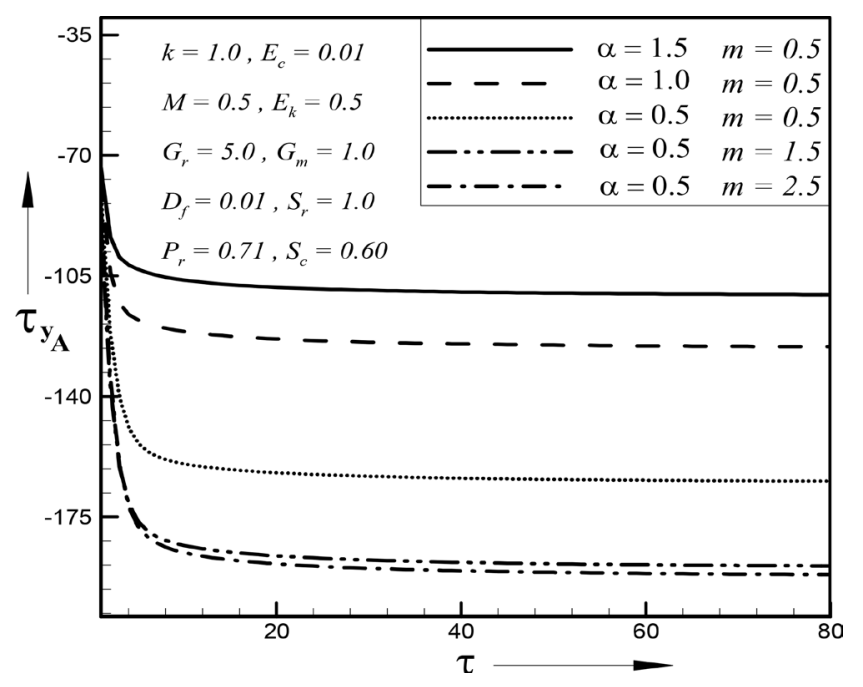

Figure 32. Average secondary shear stress for different values of $\alpha$ and $m$. 


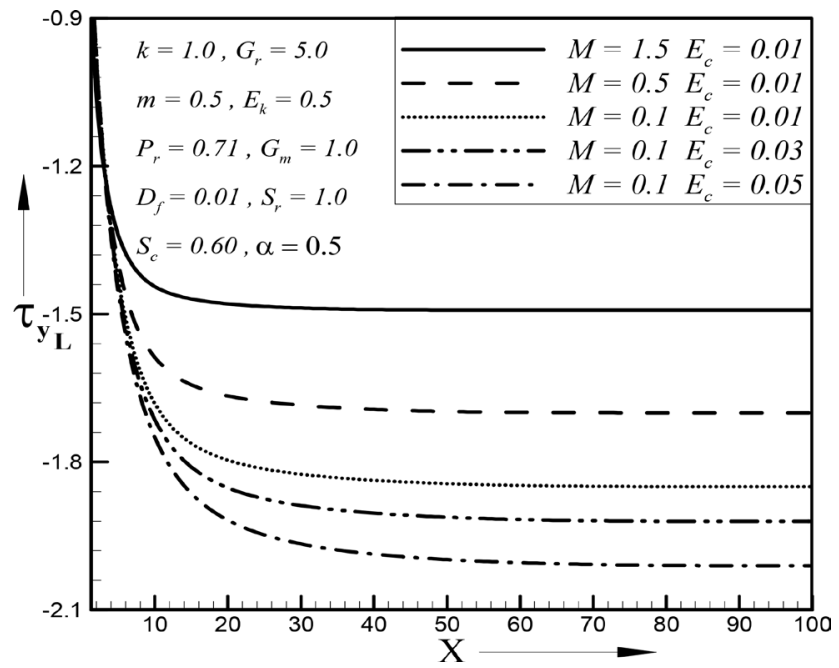

Figure 33. Local secondary shear stress for different values of $M$ and $E_{c}$.

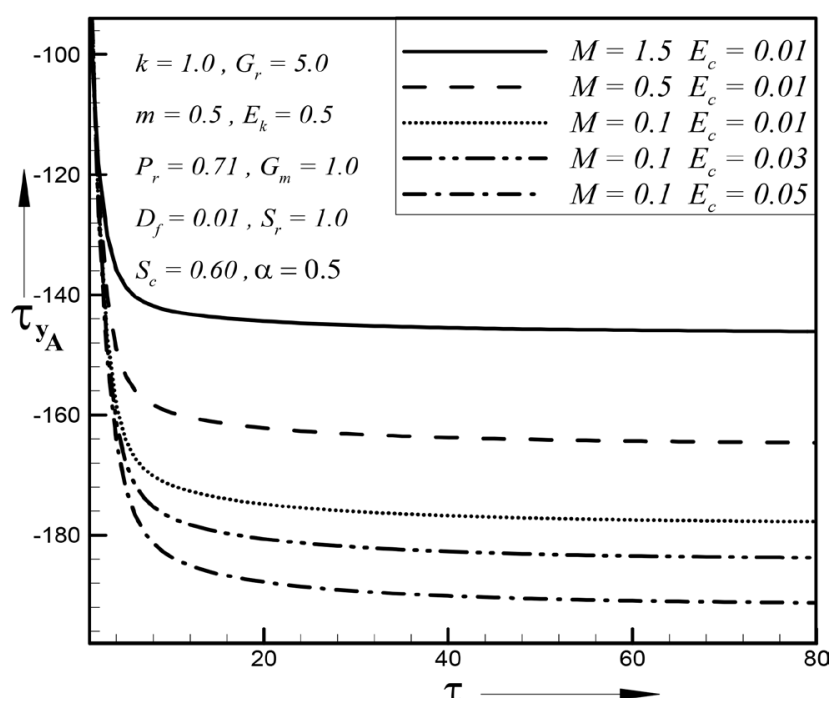

Figure 34. Average secondary shear stress for different values of $M$ and $E_{c}$.

In case of rising Dufour number $\left(D_{f}\right)$, local and average secondary shear stresses have a minor increasing effect while for the increasing values of Soret number $\left(S_{r}\right)$, they are decreasing those are observed in Figure 35 \& Figure 36.

The profiles of steady state local and average Nusselt number $N_{u_{L}}$ and $N_{u_{A}}$ for different values of parameters are shown in Figures 37-40. In Figure $37 \&$ Figure 38, $N_{u_{L}}$ and $N_{u_{A}}$ increase with the increase of Heat source parameter (a) while they have a negligible effect for Hall parameter $(m)$. In case of rising Dufour number $\left(D_{f}\right)$, local and average Nusselt number have an increasing effect while they have a decreasing effect for Soret number $\left(S_{r}\right)$, which are observed in Figure 39 \& Figure 40.

The curves of local and average Sherwood number $S_{h_{L}}$ and $S_{h_{A}}$ are illustrated in Figures 41-44. In Figure 41 \& Figure 42, local and average Sherwood number $S_{h_{L}}$ and $S_{h_{A}}$ increase with the increase of both Heat source parameter $(\alpha)$ and Schmidt number $\left(S_{c}\right)$. In case of Dufour number $\left(D_{f}\right)$, local and average 


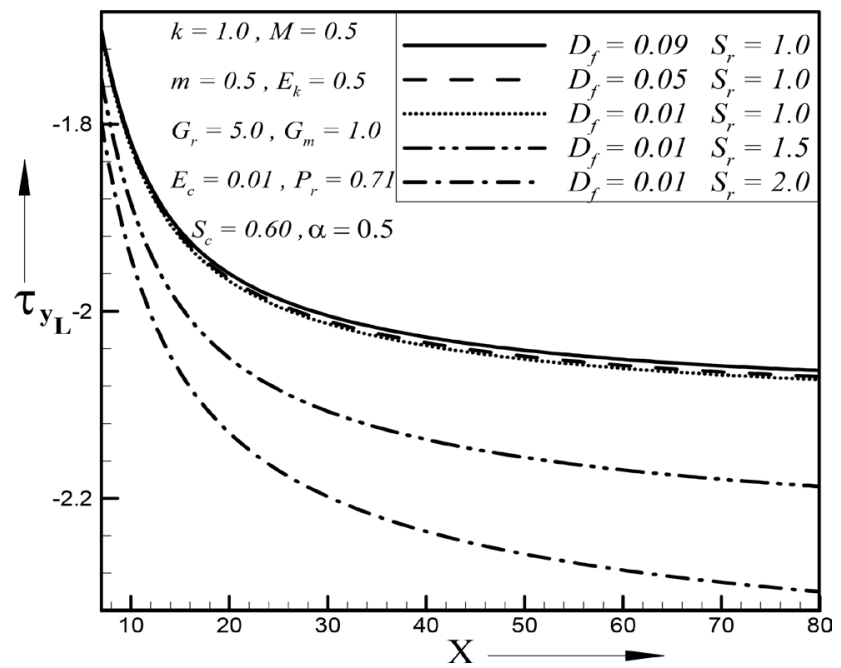

Figure 35. Local secondary shear stress for different values of $D_{f}$ and $S_{r}$.

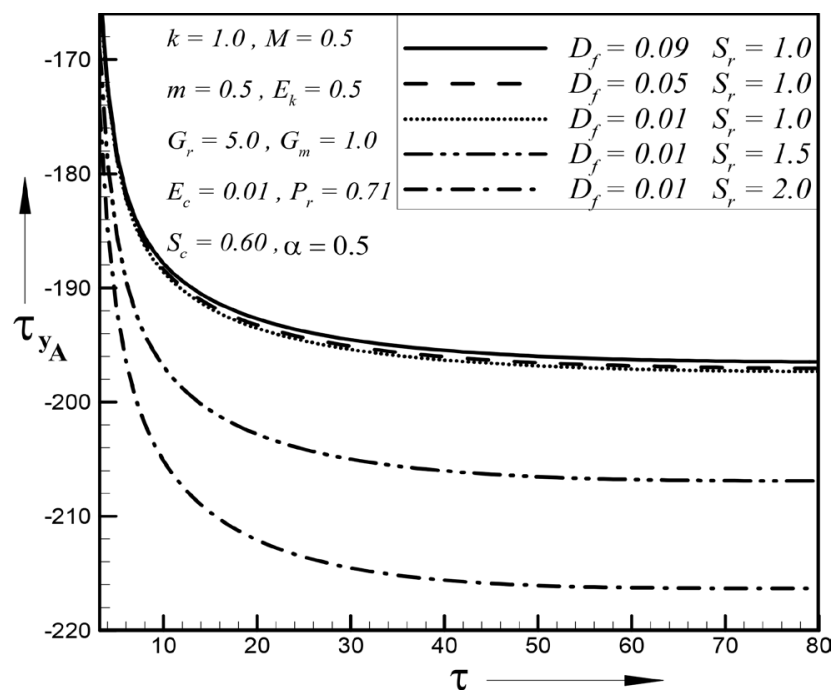

Figure 36. Average secondary shear stress for different values of $D_{f}$ and $S_{r}$.

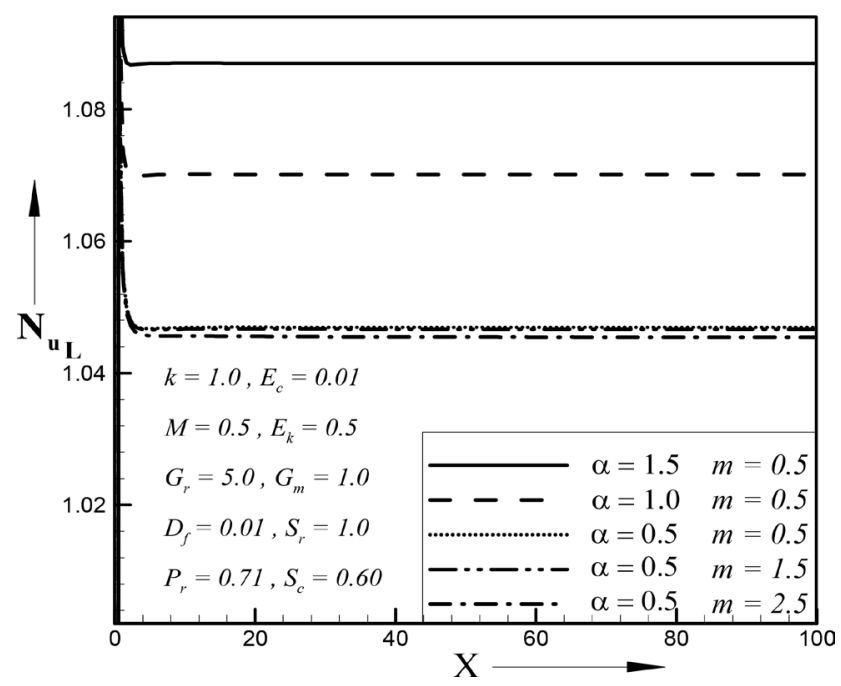

Figure 37. Local Nusselt number for different values of $\alpha$ and $m$. 


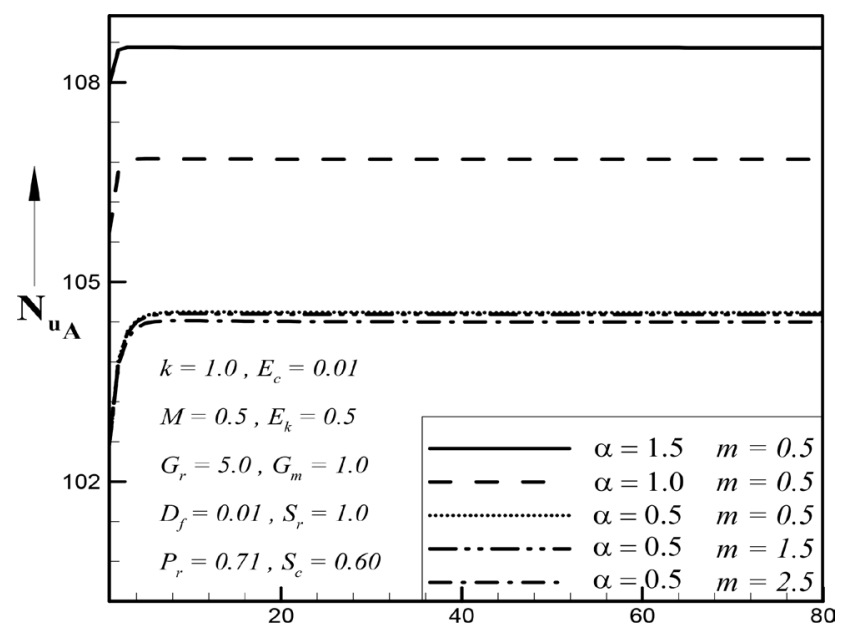

Figure 38. Average Nusselt number for different values of $\alpha$ and $m$.

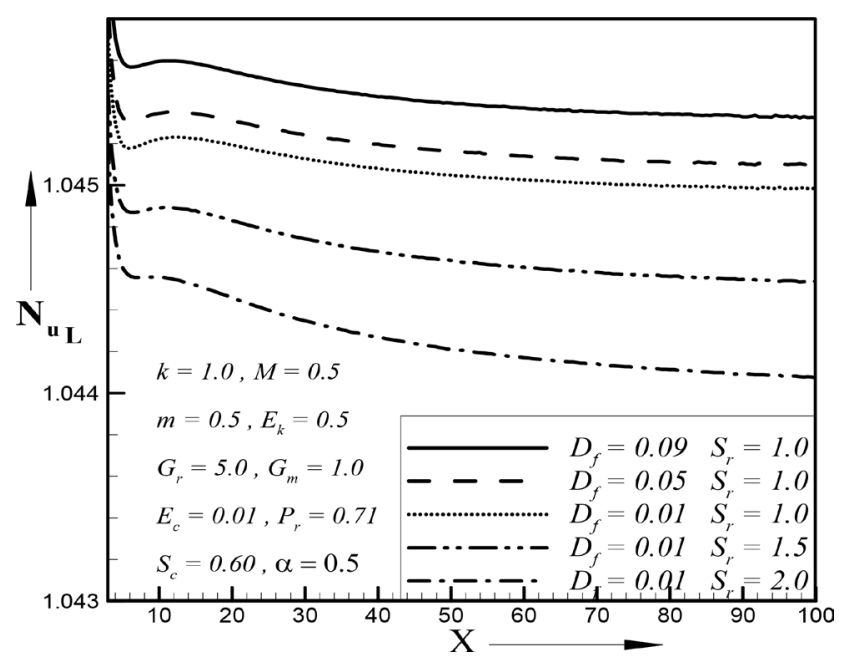

Figure 39. Local Nusselt number for different values of $D_{f}$ and $S_{r}$.

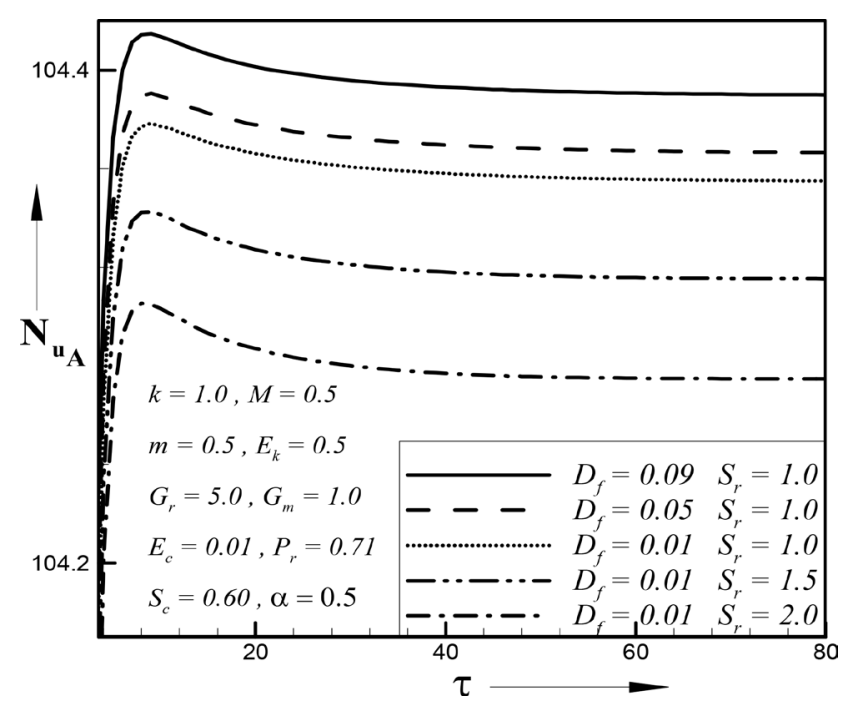

Figure 40. Average Nusselt number for different values of $D_{f}$ and $S_{r}$. 


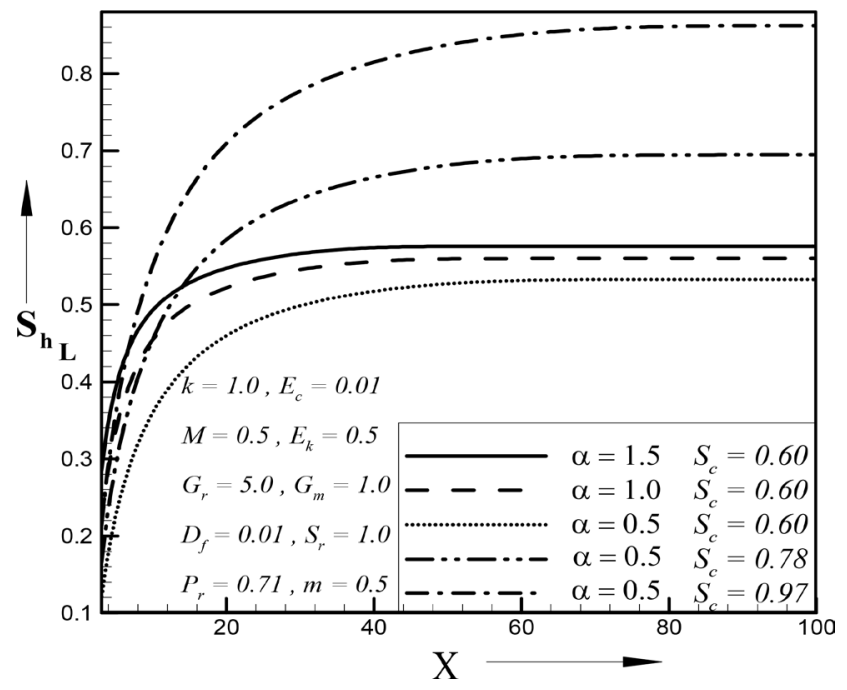

Figure 41. Local Sherwood number for different values of $\alpha$ and $S_{c \text {. }}$

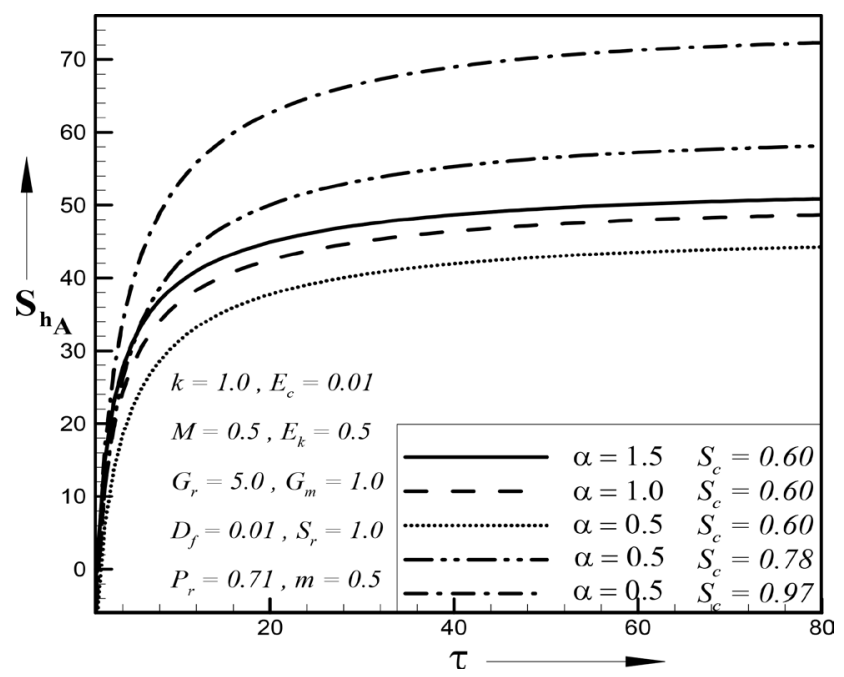

Figure 42. Average Sherwood number for different values of $\alpha$ and $S_{c}$.

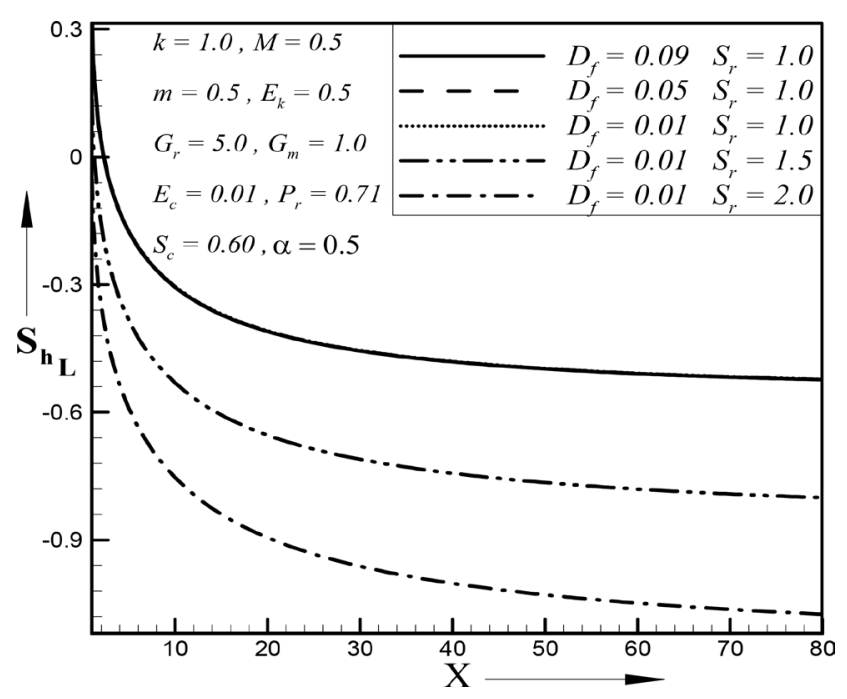

Figure 43. Local Sherwood number for different values of $D_{f}$ and $S_{r}$. 


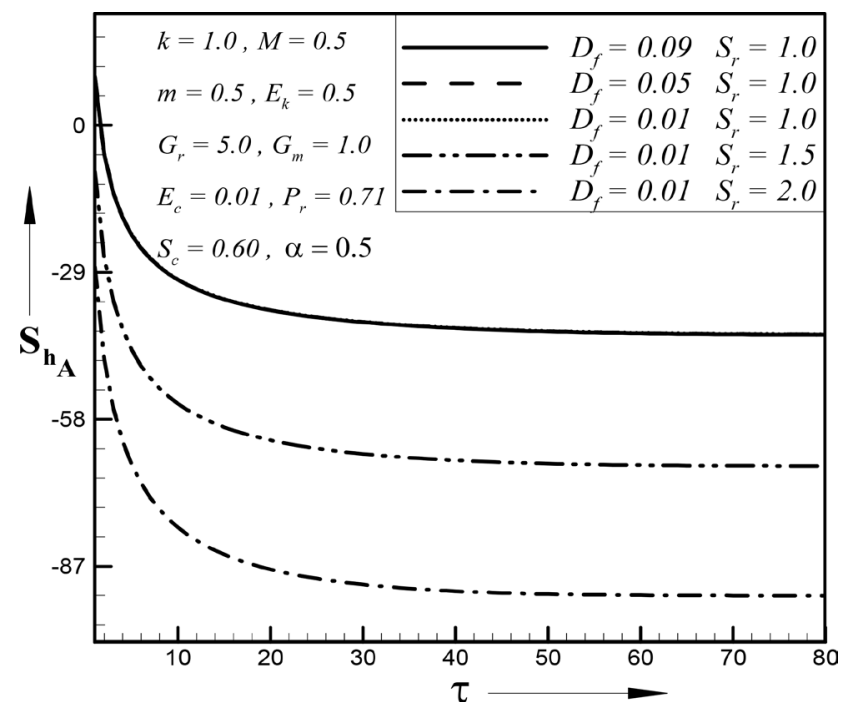

Figure 44. Average Sherwood number for different values of $D_{f}$ and $S_{r}$.

Sherwood number have a minor increasing effect while they have a decreasing effect for increasing Soret number $\left(S_{r}\right)$, which are observed in Figure 43 \& Figure 44.

\section{Conclusions}

The explicit finite difference solution for combined heat and mass transfer by free convection flow of an electrically conducting incompressible viscous fluid past an electrically non-conducting semi-infinite vertical porous plate in the presence of heat generation, joule heating, viscous dissipation, thermal diffusion and diffusion thermo with constant heat flux is investigated here. The physical properties are graphically discussed for different values of corresponding parameters. Some important findings of this investigation are below;

- The primary velocity increases with the increase of $m, E_{c}$ and $S_{r}$ while it shows reverse effect with the increase of $\alpha$ and $M$. There exists a cross flow for different values of $D_{f}$.

- With the increase of $\alpha$ and $M$, the secondary velocity increases while it decreases with the increase of $m, E_{c}$ and $S_{r}$. Also, there exists a cross flow for different values of $D_{f}$.

- The fluid temperature is increasingly affected by $E_{c}$ and decreasingly affected by $\alpha$ and $P_{r}$. A cross flow happens for different values of $D_{f}$. There is also a minor effect on temperature profile for $m$ and $S_{r}$. Particularly, the fluid temperature is more for air than water and it is less for lighter than heavier particles.

- The concentration profile is increasingly affected by $S_{r}$ and decreasingly affected by $\alpha$ and $P_{r}$. A cross flow happens for different values of $S_{c}$ and $D_{f}$ Particularly, the species concentration is higher for water than air as well as it is more for lighter than heavier particles.

- The local and average primary shear stresses increase with the increase of $m$, 
$E_{c}$ and $S_{r}$ while reverse trend arises with the increase of $\alpha, M$ and $D_{f}$

- The local and average secondary shear stress increases with the increase of $\alpha$, $M$ and $D_{f}$ while it decreases with the increase of $m, E_{c}$ and $S_{r}$.

- The local and average rate of heat transfer is increasingly affected by $\alpha$ and $D_{f}$ and decreasingly affected by $S_{r}$. There is also a negligible effect on Nusselt number for $m$.

- The local and average rate of mass transfer is increasingly affected by $\alpha$ and $S_{c}$ and decreasingly affected by $S_{r}$. There is also a negligible effect on Sherwood number for $D_{f}$

\section{Conflicts of Interest}

The authors declare no conflicts of interest regarding the publication of this paper.

\section{References}

[1] Crammer, K.P. and Pai, S.L. (1973) Magneto-Fluid Dynamic for Engineers and Applied Physicist. McGraw Hill Book Co., New York.

[2] Ferraro, V.C.A. and Plumpton, C. (1966) An Introduction to Magneto Fluid Mechanics. Clarandon Press, Oxford.

[3] Shercliff, J.A. (1965) A Text Book of Magnetohydrodynamics. Pergamon Press, London.

[4] Raptis, A.A., Perdikis, C.P. and Tzivanidis, G.J. (1981) Hydromagnetic Free Convection Flow past a Vertical Infinite Porous Plate in a Rotating Fluid. Acta Physica Academiae Scientiarum Hungaricae, 50, 373. https://doi.org/10.1007/BF03157901

[5] Ram, P.C. (1990) Effects of Hall Current and Wall Temperature Oscillation on Convective Flow in a Rotating Fluid through Porous Medium. Wärme-Stoffübertrag, 25, 205. https://doi.org/10.1007/BF01785406

[6] Kafoussias, N.G. and Williams, E.W. (1995) Thermal-Diffusion and Diffusion-Thermo Effects on Mixed Free-Forced Convective and Mass Transfer Boundary Layer Flow with Temperature Dependent Viscosity. International Journal of Engineering Science, 33, 1369-1384. https://doi.org/10.1016/0020-7225(94)00132-4

[7] Elbashbeshy, E.M.A. (1997) Heat and Mass Transfer along a Vertical Plate with Variable Surface Temperature and Concentration in the Pressure of the Magnetic Field. International Journal of Engineering Science, 34, 515-522. https://doi.org/10.1016/S0020-7225(96)00089-4

[8] Singh, A.K. (2001) MHD Free Convection and Mass Transfer Flow with Heat Source and Thermal Diffusion. Journal of Energy, Heat and Mass Transfer, 23, 227-249.

[9] Takhar, H.S., Chamkha, A.J. and Nath, G. (2002) MHD Flow over a Moving Plate in a Rotating Fluid with Magnetic Field, Hall Currents and Free-Stream Velocity. International Journal of Engineering Science, 40, 1511-1527. https://doi.org/10.1016/S0020-7225(02)00016-2

[10] Postelnicu, A. (2004) Influence of a Magnetic Field on Heat and Mass Transfer by Natural Convection from Vertical Surfaces in Porous Media Considering Soret and Dufour Effects. International Journal of Heat and Mass Transfer, 47, 1467-1472. https://doi.org/10.1016/j.ijheatmasstransfer.2003.09.017 
[11] Sharma, B.K., Jha, A.K. and Chaudhary, R.C. (2007) Hall Effect on MHD Mixed Convective Flow of a Viscous Incompressible Fluid past a Vertical Porous Plate Immersed in a Porous Medium with Heat Source/Sink. Bucharest. Romanian Journal Physics, 52, 487-505.

[12] Wabomba, M.S., Jacob, K., Kinyanjui, A.M. and Lunani, M.A. (2012) Magnetic Field and Hall Current Effect on MHD Free Convection Flow past a Vertical Rotating Flat Plate. Asian Journal of Current Engineering and Mathematics, 6, 346-354.

[13] Alam, M.M., Islam, M.R., Wahiduzzaman, M. and Rahman, F. (2012) Unsteady Heat and Mass Transfer by Mixed Convection Flow from a Vertical Porous Plate with Induced Magnetic Field, Constant Heat and Mass Fluxes. Journal of Energy, Heat and Mass Transfer, 34, 193-215.

[14] Sudhakar, K., Srinivasa Raju, R. and Rangamma, M. (2013) The Hall Effect on Unsteady MHD Flow past along a Porous Flat Plate with Thermal Diffusion, Diffusion Thermo and Chemical Reaction. International Journal of Physical and Mathematical Sciences, 4, 370-395.

[15] Shateyi, S., Mosta, S.S. and Sibanda, P. (2010) The Effects of Thermal Radiation, Hall Currents, Soret and Dufour on MHD Flow by Mixed Convection over Vertical Surface in Porous Media. Mathematical Problems in Engineering, 2010, Article ID: 627475. https://doi.org/10.1155/2010/627475

[16] Cowling, T.G. (1957) Magnetohydrodynamics. Interscience Publications, New York. 